\title{
MAGNETIC COUPLING IN THE DISKS AROUND YOUNG GAS GIANT PLANETS
}

\author{
N. J. Turner ${ }^{1}$, Man Hoi LeE ${ }^{2}$, AND T. SANO ${ }^{3}$ \\ ${ }^{1}$ Jet Propulsion Laboratory, California Institute of Technology, Pasadena, CA 91109, USA; neal.turner@jpl.nasa.gov \\ ${ }^{2}$ Department of Earth Sciences and Department of Physics, The University of Hong Kong, Pokfulam Road, Hong Kong; mhlee@hku.hk \\ ${ }^{3}$ Institute of Laser Engineering, Osaka University, Suita, Osaka 565-0871, Japan; sano@ile.osaka-u.ac.jp \\ Received 2013 June 7; accepted 2013 November 20; published 2014 February 7
}

\begin{abstract}
We examine the conditions under which the disks of gas and dust orbiting young gas giant planets are sufficiently conducting to experience turbulence driven by the magneto-rotational instability. By modeling the ionization and conductivity in the disk around proto-Jupiter, we find that turbulence is possible if the X-rays emitted near the Sun reach the planet's vicinity and either (1) the gas surface densities are in the range of the minimum-mass models constructed by augmenting Jupiter's satellites to solar composition, while dust is depleted from the disk atmosphere, or (2) the surface densities are much less, and in the range of gas-starved models fed with material from the solar nebula, but not so low that ambipolar diffusion decouples the neutral gas from the plasma. The results lend support to both minimum-mass and gas-starved models of the protojovian disk. (1) The dusty minimum-mass models have internal conductivities low enough to prevent angular momentum transfer by magnetic forces, as required for the material to remain in place while the satellites form. (2) The gas-starved models have magnetically active surface layers and a decoupled interior "dead zone." Similar active layers in the solar nebula yield accretion stresses in the range assumed in constructing the circumjovian gas-starved models. Our results also point to aspects of both classes of models that can be further developed. Non-turbulent minimum-mass models will lose dust from their atmospheres by settling, enabling gas to accrete through a thin surface layer. For the gas-starved models it is crucial to learn whether enough stellar X-ray and ultraviolet photons reach the circumjovian disk. Additionally, the stress-to-pressure ratio ought to increase with distance from the planet, likely leading to episodic accretion outbursts.
\end{abstract}

Key words: accretion, accretion disks - astrochemistry - magnetohydrodynamics (MHD) - planets and satellites: formation - turbulence

Online-only material: color figures

\section{INTRODUCTION}

Jupiter's regular satellites have nearly coplanar orbits with small eccentricities, and probably originated in an orbiting circumplanetary disk of dust and gas-a solar nebula in miniature (Lunine \& Stevenson 1982). As Jupiter approached its present mass, its tides opened a gap in the solar nebula (Lin \& Papaloizou 1986, 1993). Incoming gas then had too much angular momentum to fall directly onto the planet, and instead went into orbit, forming a circumjovian disk (Lubow et al. 1999). The disk governed the flow of material to the planet and provided the environment in which the satellites formed. A key question is therefore how quickly the orbital angular momentum was redistributed within the disk, allowing some material to accrete on the planet and some to spiral outward where it may have been removed by solar gravity or by photoevaporation. Also, was the flow laminar or turbulent? Did the released gravitational potential energy become heat in the interior, or was it dissipated in the disk atmosphere? And what did the resulting internal temperatures, densities and flow fields mean for the processing of the moon-forming ices and silicates?

As with the much larger disks orbiting young stars (LyndenBell \& Pringle 1974; Pringle 1981; Bodenheimer 1995; Armitage 2011), circumplanetary disks' evolution is controlled by the transport of orbital angular momentum. In other astrophysical disks, magnetic forces carry angular momentum outward in the turbulence resulting from magneto-rotational instability or MRI (Balbus \& Hawley 1991, 1998). The instability can work only if the disk material is ionized enough to couple to the magnetic fields. In the disks around young giant planets, as in protostellar disks, the low temperatures mean thermal ionization is ineffective except very near the central body. Ionization by radioactive isotopes' decay, lightning, bolide impacts, and planetesimal ablation is also weak (Takata \& Stevenson 1996). Adequate ionization might be produced by interstellar cosmic rays if not for rapid recombination on the surfaces of dust grains (Fujii et al. 2011). Our purpose here is to find whether the disk around Jupiter is ionized enough for MRI turbulence if an additional ionization process is considered: the X-rays from the young Sun (Igea \& Glassgold 1999). Below we compute the magnetic coupling, which depends on the ionization state, which in turn depends on the distribution of densities and temperatures.

A variety of models has been proposed for the circumjovian disk. We consider typical examples from two broad classes. In the minimum-mass models (Lunine \& Stevenson 1982; Mosqueira \& Estrada 2003a), all the ingredients for the satellites are present from an early stage. The gases are eventually dispersed while all the solids are incorporated into the satellites. The disk surface density, obtained by augmenting the rock and ice of the Galilean satellites with gases to solar or near-solar composition, is about $10^{7} \mathrm{~g} \mathrm{~cm}^{-2}$ at the surface of the planet with a power-law radial falloff. The large mass column means few cosmic rays or X-rays penetrate the interior. Recombination is rapid, and the minimum-mass disk couples poorly to magnetic fields (Takata \& Stevenson 1996).

The second class of models is gas-starved (Canup \& Ward 2002, 2006). Gas and dust trickle into the disk from the surrounding solar nebula. While some of the solids accumulate into larger solid bodies, much material is lost to the planet 
through the effective viscosity of the gas and the gravitational torques exerted by the gas on the proto-satellites. In this picture, today's moons are the last generation to form before the gas dispersed. The disk surface densities in this model are less than $1000 \mathrm{~g} \mathrm{~cm}^{-2}$, low enough that some cosmic rays can reach the midplane (Fujii et al. 2011).

While disks of gas and dust have been used to explain the moons of both Jupiter and Saturn (Sasaki et al. 2010), other classes of model may be required given that Jupiter has four large satellites with a gradient in density, while Saturn has just one large satellite. Saturn's smaller inner moons may have grown from ring particles transported out across the Roche limit, with the more distant experiencing more mergers (Crida \& Charnoz 2012). However, it is unclear whether Titan formed the same way. Another mechanism, gas-poor planetesimal capture (Safronov et al. 1986; Estrada \& Mosqueira 2006), involves collisions among a swarm of planetesimals. This picture has not so far yielded a quantitative accounting for the Galilean moons' large masses and their decrease in density with distance from the planet. We therefore focus on a circumplanetary gas and dust disk as the most promising model for the origins of Jupiter's large moons.

The paper is laid out as follows. The minimum-mass circumplanetary disk models are described in Section 2, and the gas-starved models in Section 3. The chemical reaction network used to compute the magnetic diffusivities is laid out in Section 4 and the MRI turbulence criteria in Section 5. The resulting distributions of magnetic activity in the disks are shown in Section 6. Implications for the evolution of the dust and the growth of satellites are discussed in Section 7, and our conclusions are presented in Section 8.

\section{MINIMUM-MASS CIRCUMJOVIAN DISK MODELS}

The minimum-mass models of the circumjovian disk are built in a similar way to minimum-mass solar nebula models. The satellite system's mass of $2.1 \times 10^{-4} M_{J}$ is combined with enough hydrogen and helium to reach solar composition. The resulting disk has a few percent of Jupiter's mass $M_{J}$, and extends from inside the present orbit of Io at $5.9 R_{J}$ to at least the orbit of Callisto at $26 R_{J}$ (where $R_{J}$ is the radius of Jupiter).

Temperatures in the disk's outer reaches must remain below the water sublimation threshold to account for the ice-rich makeup of Ganymede and Callisto. The release of gravitational energy as disk material spirals toward the planet may raise temperatures too high unless the accretion-stress-to-gas-pressure ratio $\alpha<10^{-5}$ (Mosqueira \& Estrada 2003a). Stresses near or above this danger level potentially arise from the damping of the wakes raised in the disk gas by satellitesimals (Goodman \& Rafikov 2001) and from the stellar tides periodically forcing the disk (Rivier et al. 2012). However, in this paper we focus on whether magnetic forces can yield still larger stresses.

Another constraint comes from observing that Callisto appears to be only partly differentiated (moment of inertia $I / M R^{2} \approx 0.355$; Anderson et al. 2001) though we note that it would be desirable to have the partly differentiated interpretation confirmed (McKinnon 1997; Gao \& Stevenson 2013). Keeping ice and rock mixed is feasible only if the ice never melted during the moon's assembly. The gravitational potential energy of the component parts must then have been released as heat over a period of 0.6 Myr or longer (Barr \& Canup 2008). To slow Callisto's growth, it may be helpful to drop the circumjovian disk's surface density sharply between Ganymede and Callisto (Mosqueira \& Estrada 2003a).
Finally, to avoid its ice melting in the heat released by short-lived radionuclide decay, Callisto also must have finished accreting at least $4 \mathrm{Myr}$ after the formation of the refractory calcium-aluminum-rich inclusions (Barr \& Canup 2008). The raw materials must persist in orbit around Jupiter until at least this date.

Each circumjovian disk model is specified by the radial profiles of gas surface density $\Sigma(r)$, solids-to-gas mass ratio $\phi(r)$ and midplane temperature $T_{c}(r)$. From these we obtain the scale height and density using

$$
H(r)=c_{s} / \Omega=\left(\frac{\mathcal{R} T_{c} r^{3}}{\mu G M_{J}}\right)^{1 / 2}
$$

and

$$
\rho(r, z)=\frac{\Sigma(r)}{\sqrt{2 \pi} H} \exp \left[-z^{2} /\left(2 H^{2}\right)\right],
$$

where $c_{s}$ is the isothermal sound speed, $\Omega$ the orbital frequency, $\mathcal{R}$ the gas constant, and $\mu=2.3$ the mean molecular weight, and the density varies with the cylindrical coordinates $(r, z)$.

For each model we consider versions in which the solids (1) take the form of sub-micron dust grains, and (2) are locked up in bodies of $1 \mathrm{~cm}$ or larger. Particles this big are few enough that their combined cross-section for recombination is too low to affect the abundances of free charges. We set the sub-micron grains' dust-to-gas mass ratio $\epsilon(r)$ equal to $\phi(r)$ in the first, dusty case and zero in the second, dust-free case.

\subsection{Takata \& Stevenson (1996) Model-MM96}

We include a minimum-mass model very similar to that used by Takata \& Stevenson (1996) to facilitate comparison with their ionization results. This model, which we call MM96, has the simple surface density profile

$$
\Sigma(r)=\Sigma_{0}\left(R_{J} / r\right)
$$

with $\Sigma_{0}=10^{7} \mathrm{~g} \mathrm{~cm}^{-2}$, and the temperature profile

$$
\begin{aligned}
T_{c}(r) & =3600\left(R_{J} / r\right) \mathrm{K} \text { for } r / R_{J} \leqslant 30 \\
& =120 \mathrm{~K} \text { for } r / R_{J} \geqslant 30 .
\end{aligned}
$$

The model differs from Takata \& Stevenson (1996) in that we compute the density scale height from the temperature via Equation (1), yielding $H=0.086 r$ within $30 R_{J}$ and $H \sim r^{3 / 2}$ beyond, while they simply took $\sqrt{2} H \approx 0.1 r$. We have checked that the two density distributions yield similar magnetic diffusivities under X-ray ionization and dust surface recombination. Our MM96 model includes a 1\% mass fraction of solid material.

The surface density and midplane temperature profiles of the MM96 model are plotted in Figure 1 along with those of the six other models described below.

\subsection{Mosqueira \& Estrada (2003a) Model-MM03}

The MM03 model has a more complex surface density profile,

$$
\begin{aligned}
\Sigma(r) & =\Sigma_{\text {in }}^{0}\left(R_{\text {in }} / r\right) \quad \text { for } r \leqslant r_{1} \\
& =a_{1}\left(r_{1} / r\right)^{b_{1}} \quad \text { for } r_{1} \leqslant r \leqslant r_{2} \\
& =\Sigma_{\text {out }}^{0}\left(R_{\text {out }} / r\right) \quad \text { for } r \geqslant r_{2}
\end{aligned}
$$



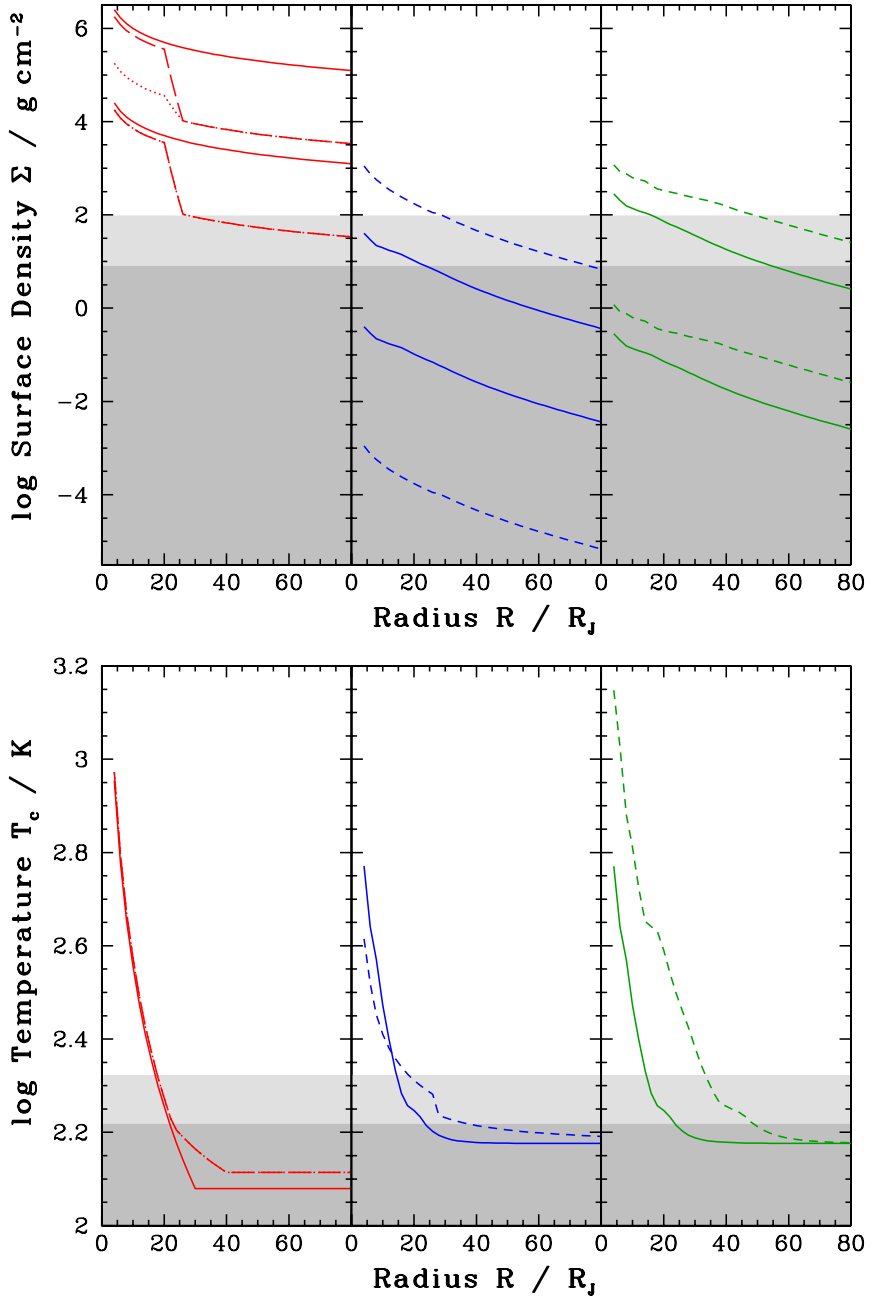

Figure 1. Seven subnebula models' radial profiles of surface density (top) and midplane temperature (bottom). The minimum-mass models appear at left, the gas-starved models in the center and right panels. Each model's gas and dust surface densities are shown by two matching curves, with the gas the larger one. The three minimum-mass models at left are MM96 (solid), MM03 (longdashed), and SEMM (dotted). Note that the MM03 and SEMM models overlap throughout in solid surface density and temperature. The gas-starved models at center differ in opacity and are $\mathrm{K} 0$ (solid) and K-4 (dashed), while those at right differ in the growth timescale and are TG50 (solid) and TG05 (dashed). Gray shading in the surface density panels indicates the range penetrated by Xrays (darker) and cosmic rays (lighter). The shadings in the temperature panels indicate the approximate water ice stability range at the minimum (darker) and maximum (lighter) pressures found in the gas-starved models (Canup \& Ward 2002).

(A color version of this figure is available in the online journal.)

where $\Sigma_{\text {in }}^{0}=51 \times 10^{4} \mathrm{~g} \mathrm{~cm}^{-2}, \Sigma_{\text {out }}^{0}=0.31 \times 10^{4} \mathrm{~g} \mathrm{~cm}^{-2}$, $r_{1}=20 R_{J}, r_{2}=26 R_{J}, R_{\text {in }}=14 R_{J}, R_{\text {out }}=87 R_{J}$,

$$
a_{1}=\Sigma_{\text {in }}^{0} R_{\text {in }} / r_{1}=35.7 \times 10^{4} \mathrm{~g} \mathrm{~cm}^{-2},
$$

and

$$
b_{1}=\ln \left[\left(\Sigma_{\text {in }}^{0} R_{\text {in }} r_{2}\right) /\left(\Sigma_{\text {out }}^{0} R_{\text {out }} r_{1}\right)\right] / \ln \left(r_{2} / r_{1}\right)=13.4871
$$

Note that the values of $a_{1}$ and $b_{1}$ in Table 2 of Mosqueira \& Estrada (2003a) are not exact and that there is a missing $r_{1}$ in their Equation (6) for $\Sigma$ in the transition region.
The temperature in Kelvins, based on fitting their Figure 3, is

$$
\begin{aligned}
T_{c}(r) & =3750\left(R_{J} / r\right) \quad \text { for } r / R_{J} \leqslant 23 \\
& =655.55\left(r / R_{J}\right)^{-1 / 2}+26.35 \text { for } 23 \leqslant r / R_{J} \leqslant 40 \\
& =130 \text { for } r / R_{J} \geqslant 40 .
\end{aligned}
$$

Like MM96, our MM03 model includes a 1\% mass fraction of solid material.

\subsection{Solids-enhanced Minimum-mass Model-SEMM}

The solids-enhanced minimum-mass model preferred by Mosqueira \& Estrada (2003b) and Estrada et al. (2009) differs from the MM03 model in having $90 \%$ of the gas removed within $r_{1}=20 R_{J}$. The gas surface density is unchanged outside $r_{2}=26 R_{J}$, and in the transition zone between 20 and $26 R_{J}$ varies smoothly as $3.57 \times 10^{4}\left(r / r_{1}\right)^{-4.711} \mathrm{~g} \mathrm{~cm}^{-2}$. The surface density of the solids is left unchanged throughout. In this sense, the model is not solids-enhanced but gas-depleted.

\section{IMPROVED GAS-STARVED SUBNEBULA MODEL}

In the gas-starved models, only a fraction of the material needed to form the satellites orbits the planet at any given instant. The subnebula is replenished by the slow inflow of gas and solids after Jupiter opens a gap in the solar nebula. An approximate overall balance between the growth of new satellites and loss by migrating into the planet regulates the mass fraction of the satellite system to $\sim 10^{-4}$ (Canup \& Ward 2006).

Gas-starved models are constructed assuming material from the solar nebula falls steadily on the circumplanetary disk (Canup \& Ward 2002). Hydrodynamical calculations treating the vertical structure show the solar nebula gas approaches the planet and its disk from above and below (Machida et al. 2008; Tanigawa et al. 2012; Ayliffe \& Bate 2012). The circumplanetary disk structure is insensitive to the distribution of the injected solar nebula gas once a steady-state is reached, depending instead on the disk's angular momentum balance (Martin \& Lubow 2011). This contrasts with the minimum-mass models, where the size is fixed by the angular momentum of the gas at the time the disk is assembled. Orbital angular momentum is transferred through the gas-starved subnebula by an unspecified process that yields accretion stresses equal to a constant, $\alpha$, times the gas pressure (Shakura \& Sunyaev 1973). The temperature is determined by the resulting release of gravitational energy, together with the illumination from Jupiter and from the surrounding solar nebula, balanced by radiative losses.

Regarding the circumplanetary disk's size, we can say that the outer edge lies within $40 \%$ of the planet's Hill radius $r_{H}$, since at greater distances the stellar tide is strong and periodic ballistic orbits cross (Martin \& Lubow 2011). A smaller disk can expand to $0.4 r_{H}$ under magnetic stresses (Lubow \& Martin 2013). On the other hand, photoevaporation is capable of truncating circumplanetary disks to a small fraction of the Hill radius (Mitchell \& Stewart 2011). The maximum size of $0.4 r_{H}$ for Jupiter corresponds to about $300 R_{J}$.

Several further constraints apply to conditions inside Jupiter's disk. Ganymede's composition requires the water ice sublimation point to lie inside this moon's orbit when the last generation of satellites form. Slow growth of the planet before the solar nebula starts to dissipate is ruled out because the stellar tides raise a two-armed spiral wave in hydrodynamical models of an inviscid circumplanetary disk, setting a floor on the accretion torques that yields a slowest allowed planet mass doubling 
time of 5 Myr (Rivier et al. 2012). This suggests the maximum $\tau_{G}=10^{8}$ yr used by Canup \& Ward (2002) applies only after the solar nebula starts to dissipate. Yet another constraint comes from Alibert et al. (2005) who found that rocky satellites within $10 R_{J}$ survive migration if $\alpha>2 \times 10^{-4}$ and temperatures remain low enough for long enough to form Callisto if $\alpha<10^{-3}$. Note that the heating in their models is distributed in the disk interior. Releasing the heat in a magnetically active surface layer at lower optical depth yields cooler midplane temperatures (Hirose \& Turner 2011). We therefore do not attempt to meet the last constraint.

In the gas-starved models of Canup \& Ward (2002), the inflowing material is assumed to be deposited uniformly in the region extending to distance $r_{c}$ from the planet, with the total rate of mass inflow equal to $F_{*}$. The gas component of the disk spreads viscously, both onto the planet and out to some assumed outer edge at $r_{d}$. Three parameters distinguish the gas-starved models of Canup \& Ward (2002). These are the stress-to-pressure ratio $\alpha$; the opacity of the disk to its own radiation, assumed independent of temperature, density and position; and the rate at which mass falls on the planet, measured by the planet growth timescale $\tau_{G}=M_{J} / \dot{M}_{J}$ (where $\dot{M}_{J} \approx F_{*}$ ). We construct versions of these models with three improvements, (1) making the opacities temperature-dependent, (2) properly treating optically thin disk annuli, and (3) more accurately computing the illumination by Jupiter. Due to the first of these, we replace their constant opacity parameter $K$ by a dust depletion factor, for which we use the symbol $f_{\text {opac }}=\epsilon / 0.01$. The temperature-dependent opacities are taken from Pollack et al. (1994). The second and third improvements are made by using midplane temperatures from the analytic vertical structure model of Hubeny (1990) for viscous dissipation and isotropic solar nebula irradiation, with the extension for irradiation by a central source (i.e., Jupiter) by Malbet et al. (2001).

The temperature-dependent opacity for undepleted grain composition is taken from Figure 6 of Pollack et al. (1994), which includes contributions from silicates, troilite, metallic iron, organics, and water ice. It increases from $0 \mathrm{~cm}^{2} \mathrm{~g}^{-1}$ at $0 \mathrm{~K}$ to $6.50 \mathrm{~cm}^{2} \mathrm{~g}^{-1}$ at $174 \mathrm{~K}$, and shows multiple local minima and maxima at higher temperatures, ranging from $1.95 \mathrm{~cm}^{2} \mathrm{~g}^{-1}$ at $700 \mathrm{~K}$ to $6.28 \mathrm{~cm}^{2} \mathrm{~g}^{-1}$ at $425 \mathrm{~K}$.

The product of the disk gas surface density $\Sigma$ and viscosity $v$ is determined by the mass inflow model and independent of the vertical structure. So

$$
\nu \Sigma=\frac{4 F_{*}}{15 \pi} \begin{cases}\frac{5}{4}-\sqrt{\frac{r_{c}}{r_{d}}}-\frac{1}{4}\left(\frac{r}{r_{c}}\right)^{2} & \text { for } r<r_{c} \\ \sqrt{\frac{r_{c}}{r}}-\sqrt{\frac{r_{c}}{r_{d}}} & \text { for } r \geqslant r_{c}\end{cases}
$$

as in Canup \& Ward (2002). We also follow Canup \& Ward (2002) in adopting the $\alpha$ prescription for the viscosity:

$$
v=\alpha c_{s}^{2} / \Omega
$$

where $c_{s}$ is the midplane sound speed.

We consider heating by viscous dissipation in the disk, incoming isotropic radiation at the ambient nebular temperature $T_{\text {neb}}$, and incoming radiation from Jupiter. According to orderof-magnitude estimates, radial heat advection is unimportant.

The irradiation from our central source, Jupiter, is highly directional, with the cosine of the characteristic angle (measured from the inward directed normal to the disk surface) at which the light enters the disk equal to (Chiang \& Goldreich 1997)

$$
\mu_{J}=\frac{4}{3 \pi}\left(\frac{R_{J}}{r}\right)+\left(\frac{H}{r}\right)\left(\frac{d \ln H}{d \ln r}-1\right),
$$

and the flux intercepted by a surface element (either top or bottom) of the disk equal to

$$
4 \pi H_{J}(0)=\left(\frac{\mu_{J}}{2}\right)\left(\frac{R_{J}}{r}\right)^{2} \sigma_{\mathrm{SB}} T_{J}^{4},
$$

where $\sigma_{\mathrm{SB}}$ is the Stefan-Boltzmann constant. Equation (17) was derived by Ruden \& Pollack (1991) in the limit that $H / r \ll 1$ and $r \gg R_{J}$ (see also Kusaka et al. 1970). It differs from $4 \pi H_{J}(0)=(d H / d r)\left(R_{J} / r\right)^{2} \sigma_{\mathrm{SB}} T_{J}^{4}$ used by Canup \& Ward (2002).

Energy balance requires the outgoing flux on the disk surface to equal to the sum of the incoming flux plus the emission from viscous dissipation. Thus the net (outgoing minus incoming) flux on the surface of the disk is just the emission from viscous dissipation. If we define the accretion temperature $T_{d}$ in terms of the net flux,

$$
2 \sigma_{\mathrm{SB}} T_{d}^{4}=\frac{9}{4} \Omega^{2} v \Sigma .
$$

To determine the midplane and surface temperatures, we use the analytic model of the vertical structure developed by Hubeny (1990) for the treatment of viscous dissipation and isotropic irradiation by the ambient nebula, and we use the extension of this model by Malbet et al. (2001) for the treatment of irradiation by a central source (Jupiter in our case). We use the simplest form of this model with the following approximations. We assume that the different forms of mean opacities are all equal to the Rosseland mean opacity. We use the same mean opacity for the disk's own radiation, the radiation from the ambient nebula, and the radiation from Jupiter. We assume that the extinction is dominated by absorption. Then the temperature at optical depth $\tau$ from the surface is given by (see Equation (3.11) of Hubeny 1990 and Equation (61) of Malbet et al. 2001)

$$
\begin{aligned}
T^{4}(\tau)= & \frac{3}{4}\left[\tau\left(1-\frac{\tau}{2 \tau_{c}}\right)+\frac{1}{\sqrt{3}}+\frac{1}{3 \tau_{c}}\right] T_{d}^{4}+T_{\mathrm{neb}}^{4} \\
& +\frac{3}{4}\left[\mu_{J}\left(1-e^{-\tau / \mu_{J}}\right)+\frac{1}{\sqrt{3}}+\frac{1}{3 \mu_{J}} e^{-\tau / \mu_{J}}\right] \\
& \times\left(\frac{4 \pi H_{J}(0)}{\sigma_{\mathrm{SB}}}\right),
\end{aligned}
$$

where $\tau_{c}$ is the optical depth to the midplane:

$$
\tau_{c}=\kappa \Sigma / 2
$$

and we use for $\kappa$ the Rosseland mean opacity at the midplane temperature. From Equation (19), the midplane temperature (at $\tau=\tau_{c}$ ) is given by

$$
\begin{aligned}
T_{c}^{4}= & \frac{3}{4}\left[\frac{\tau_{c}}{2}+\frac{1}{\sqrt{3}}+\frac{1}{3 \tau_{c}}\right] T_{d}^{4}+T_{\mathrm{neb}}^{4} \\
& +\frac{3}{4}\left[\mu_{J}\left(1-e^{-\tau_{c} / \mu_{J}}\right)+\frac{1}{\sqrt{3}}+\frac{1}{3 \mu_{J}} e^{-\tau_{c} / \mu_{J}}\right] \\
& \times\left(\frac{\mu_{J}}{2}\right)\left(\frac{R_{J}}{r}\right)^{2} T_{J}^{4},
\end{aligned}
$$


while the surface temperature (at $\tau=0$ ) is given by

$$
T_{s}^{4}=\frac{3}{4}\left[\frac{1}{\sqrt{3}}+\frac{1}{3 \tau_{c}}\right] T_{d}^{4}+T_{\mathrm{neb}}^{4}+\frac{1}{8}\left(\frac{R_{J}}{r}\right)^{2} T_{J}^{4}
$$

for $\mu_{J} \ll 1$. The term in Equation (21) with $T_{d}^{4}$ due to viscous heating differs from the expression used by Canup \& Ward (2002) in the numerical coefficients and especially in the $1 / \tau_{c}$ dependence in the optically thin limit $\left(\tau_{c} \ll 1\right)$. Canup \& Ward (2009) corrected the Canup \& Ward (2002) expression for viscous heating for the optically thin regime, but their expression also differs from Equation (21) in the numerical coefficients.

If $\tau_{c} \ll \mu_{J} \ll 1$,

$$
T_{c}^{4} \approx T_{s}^{4} \approx \frac{1}{4 \tau_{c}} T_{d}^{4}+T_{\mathrm{neb}}^{4}+\frac{1}{8}\left(\frac{R_{J}}{r}\right)^{2} T_{J}^{4} .
$$

If $\tau_{c} \ll 1$ but $\tau_{c} \ll \mu_{J}$, the last term of Equation (21) cannot be reduced to $(1 / 8)\left(R_{J} / r\right)^{2} T_{J}^{4}$. If $\tau_{c} \gg 1$,

$$
T_{c}^{4} \approx \frac{3 \tau_{c}}{8} T_{d}^{4}+T_{\mathrm{neb}}^{4}+\frac{\sqrt{3}}{4}\left(\frac{\mu_{J}}{2}\right)\left(\frac{R_{J}}{r}\right)^{2} T_{J}^{4},
$$

and

$$
T_{s}^{4} \approx \frac{\sqrt{3}}{4} T_{d}^{4}+T_{\mathrm{neb}}^{4}+\frac{1}{8}\left(\frac{R_{J}}{r}\right)^{2} T_{J}^{4} .
$$

We consider four gas-starved models, two of which are similar to the high $\left(K=1 \mathrm{~cm}^{2} \mathrm{~g}^{-1}\right)$ and low $\left(K=10^{-4} \mathrm{~cm}^{2} \mathrm{~g}^{-1}\right)$ opacity models considered by Canup \& Ward (2002). For the $f_{\text {opac }}=1$ model, which is optically thick out to $\sim 60 R_{J}$, we only need to decrease $\tau_{G}$ slightly to produce surface density and midplane temperature profiles that are similar to those shown in Figure 6 of Canup \& Ward (2002). For the $f_{\text {opac }}=10^{-4}$ model, almost the entire subnebula is optically thin, and we obtain significantly higher midplane temperatures with our improved vertical structure model (which radiates away the accretion power inefficiently in the optically thin regime), if we take the parameters from Figure 5 of Canup \& Ward (2002). To place the ice sublimation front near Ganymede's orbit, we therefore use a lower stress parameter $\alpha$ and a longer growth timescale $\tau_{G}$. Since the improved and Canup \& Ward (2002) models have similar surface density and midplane temperature profiles after suitably adjusting $\alpha$ and $\tau_{G}$, the models are expected to have similar ionization states. However, the satellites' orbital migration as they interact with the disk can be very different in the improved models, due to sharp jumps in the local power-law indices of the surface density and midplane temperature profiles resulting from the temperature-dependent opacity (Li \& Lee 2013).

In addition, we consider a scenario where the total rate of mass inflow to the disk is initially nearly constant at $F_{*}(0)$ and then decays exponentially with time, i.e., $F_{*}(t)=F_{*}(0) \exp \left(-t / \tau_{\text {in }}\right)$, due to the dispersal of the solar nebula (Canup \& Ward 2006; Barr \& Canup 2008; Ogihara \& Ida 2012). During the exponential decay, the total mass delivered to the disk after time $t$ is $F_{*}(t) \tau_{\text {in }}$. The last generation of satellites has total mass $M_{T}$ and forms after time $t_{s}$, where $F_{*}\left(t_{s}\right) \tau_{\text {in }}=M_{T} / \Phi$ and the overall solids-to-gas ratio in the inflow $\Phi \approx 0.01$. Note that $\Phi$ is a surface-integrated measure of the infalling material and need not match $\phi(r)$, the disk's internal radial profile. So $\tau_{G}\left(t_{s}\right)=M_{J} / F_{*}\left(t_{s}\right)=\left(M_{J} \Phi / M_{T}\right) \tau_{\text {in }}$. For the Jovian satellites, if $\tau_{\text {in }} \approx 1 \mathrm{Myr}, \tau_{G}\left(t_{s}\right) \approx 50 \mathrm{Myr}$. Thus we consider two models with $\tau_{G}(0)=5 \mathrm{Myr}$ and $\tau_{G}\left(t_{s}\right)=50 \mathrm{Myr}$.
Table 1

The Four Gas-starved Subnebula Models

\begin{tabular}{llcc}
\hline \hline Name & $\alpha$ & $\begin{array}{c}\tau_{G} \\
(\mathrm{Myr})\end{array}$ & $f_{\text {opac }}$ \\
\hline K0 & 0.005 & 70 & 1 \\
K-4 & 0.0009 & 20 & $10^{-4}$ \\
TG05 & 0.001 & 5 & 0.1 \\
TG50 & 0.001 & 50 & 0.1 \\
\hline
\end{tabular}

The four specific gas-starved models we construct have the parameters listed in Table 1 , as well as $r_{c}=30 R_{J}, r_{d}=150 R_{J}$, $T_{\text {neb }}=150 \mathrm{~K}$, and $T_{J}=500 \mathrm{~K}$, and their surface density and midplane temperature profiles are shown in Figure 1. Treating the opacities' temperature dependence leads to several new features. For example, the steep $T_{c}$ drop near $27 R_{J}$ in the K-4 model results from a sharp decline in the opacity at $174 \mathrm{~K}$ where water ice sublimates. The model is marginally opticallythin at this location. Temperatures are significantly higher in the $\tau_{G}(0)=5 \mathrm{Myr}$ model, which is allowed as there are no compositional constraints on the earlier generations of satellites lost by migration into the planets.

\section{IONIZATION STATE}

\subsection{Chemical Network}

The ionization state is calculated by integrating a chemical network treating in simplified form the most important gasphase pathways: molecular ionization, dissociative molecular recombination, charge transfer to metal atoms, and radiative recombination of metal ions. The closed and balanced set of reactions related to the representative molecular ion $\mathrm{HCO}^{+}$is

$$
\begin{gathered}
\mathrm{H}_{2}+\mathrm{X} \rightarrow \mathrm{H}_{2}^{+}+e^{-} \\
\mathrm{H}_{2}^{+}+\mathrm{H}_{2} \rightarrow \mathrm{H}_{3}^{+}+\mathrm{H} \\
\mathrm{H}_{3}^{+}+\mathrm{CO} \rightarrow \mathrm{HCO}^{+}+\mathrm{H}_{2} \\
2 \mathrm{H}+G \rightarrow \mathrm{H}_{2}+G \\
\mathrm{HCO}^{+}+e^{-} \rightarrow \mathrm{CO}+\mathrm{H} .
\end{gathered}
$$

Here every species (except the $X$-rays, $X$ and grains, $G$ ) is created in at least one reaction and destroyed in at least one other. Over the whole set, no species is produced or consumed on balance.

The subset producing the ions and electrons, Equations (26)(29), boils down to

$$
2 \mathrm{H}_{2}+2 \mathrm{X}+2 \mathrm{CO} \rightarrow \mathrm{H}_{2}+2 \mathrm{HCO}^{+}+2 e^{-} \text {. }
$$

That is, each X-ray striking a hydrogen molecule yields one ion and one electron. We follow Ilgner \& Nelson (2006) and others in approximating Equations (31) and (30) by

$$
\begin{gathered}
\mathrm{H}_{2}+X \rightarrow \mathrm{HCO}^{+}+e^{-} \\
\mathrm{HCO}^{+}+e^{-} \rightarrow \mathrm{H}_{2},
\end{gathered}
$$

neglecting the fact that the molecular ion holds just one hydrogen atom. This is fine since the $\mathrm{HCO}^{+}$is orders of magnitude less abundant than the $\mathrm{H}_{2}$ and forming the ions leaves the $\mathrm{H}_{2}$ density 
basically unchanged. Similarly, we do not follow CO destruction and reformation since the ion is so much less abundant than the molecule. To Equations (32) and (33) we add the charge transfer and radiative recombination reactions involving the representative metal magnesium:

$$
\begin{gathered}
\mathrm{HCO}^{+}+\mathrm{Mg} \rightarrow \mathrm{HCO}+\mathrm{Mg}^{+} \\
\mathrm{Mg}^{+}+e^{-} \rightarrow \mathrm{Mg}+h v .
\end{gathered}
$$

The product radical in Equation (34) readily breaks apart into $\mathrm{H}$ and $\mathrm{CO}$. Simplifying by again taking into account the large abundances of $\mathrm{H}_{2}$ relative to $\mathrm{H}$, and $\mathrm{CO}$ relative to $\mathrm{HCO}^{+}$, we arrive at the reduced network

$$
\begin{gathered}
\mathrm{H}_{2}+X \rightarrow \mathrm{HCO}^{+}+e^{-} \\
\mathrm{HCO}^{+}+e^{-} \rightarrow \mathrm{H}_{2} \\
\mathrm{HCO}^{+}+\mathrm{Mg} \rightarrow \mathrm{H}_{2}+\mathrm{Mg}^{+} \\
\mathrm{Mg}^{+}+e^{-} \rightarrow \mathrm{Mg}+h v .
\end{gathered}
$$

Whether the carbon is oxidized or reduced makes little difference here because (1) the rate coefficient for pathway 37 at $3 \times 10^{-6} / \sqrt{T} \mathrm{~cm}^{3} \mathrm{~s}^{-1}$ (Ilgner \& Nelson 2006) is similar to that for the corresponding methane ion, $10^{-6}$ (Takata \& Stevenson 1996), and (2) anyway gas-phase recombination proves less important than the grain surface pathway, near the dead zone boundary in our cases with dust.

Also treated in the network are grain charging and discharging (Ilgner \& Nelson 2006) through collisions with ions and electrons, and charge exchange in grain-grain collisions. Grain charges from -2 to +2 are considered. Additionally, the metal atoms are allowed to thermally adsorb on and desorb from the grains. The reactions and their rate coefficients are described by Ilgner \& Nelson (2006), with the electron sticking probabilities revised to include the grain charge following Bai (2011). The magnesium locked up inside grains is assumed to be $99 \%$ of the solar abundance of $3.7 \times 10^{-5}$ per hydrogen atom, with the remaining $1 \%$ available to participate in the recombination network, either in the gas phase or adsorbed on grain surfaces. The gas-phase magnesium abundance had little effect on the magnetic activity above a threshold level of $10^{-6}$ times solar, in protostellar disk models by Turner et al. (2007). We solve the kinetic equations describing the reaction network using a semiimplicit extrapolation method. While bringing the network to equilibrium we record the recombination time $t_{\text {rec }}$ needed to reach an electron fraction within $1 \%$ of the equilibrium value.

We include monodisperse grains $a=0.1 \mu \mathrm{m}$ in radius with internal density $\rho_{d}=2 \mathrm{~g} \mathrm{~cm}^{-3}$. This yields a geometric crosssection per unit dust mass similar to that of the size distribution used to compute the opacities by Pollack et al. (1985) and Pollack et al. (1994). Furthermore, the same dust-to-gas ratios are used for the opacities and the grain surface recombination in the dusty versions of our gas-starved models.

\subsection{Ionization Processes}

The chemical reaction network is driven by the ionization from interstellar cosmic rays, radioisotope decay, and protosolar $\mathrm{X}$-rays. The cosmic rays yield an ionization rate $10^{-17} \mathrm{~s}^{-1}$ well outside the solar nebula. They strike our material isotropically

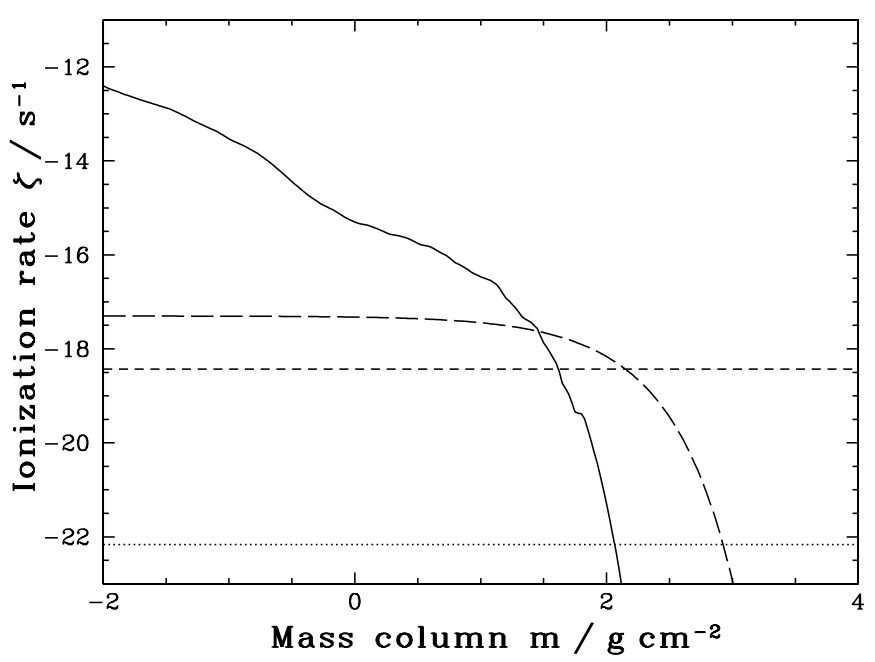

Figure 2. Ionization rates per $\mathrm{H}$ nucleus vs. the column of overlying material, resulting from X-rays (solid curve), cosmic rays (long-dashed curve), and shortand long-lived radionuclides (dashed and dotted lines). Only the X-rays and cosmic rays arriving from above are included. The X-ray ionization rate is from Monte Carlo calculations of the transfer through the solar nebula of photons with a $5 \mathrm{keV}$ thermal spectrum (Igea \& Glassgold 1999). The X-rays are extrapolated past $80 \mathrm{~g} \mathrm{~cm}^{-2}$ using the $e$-folding depth of their scattered component, $8 \mathrm{~g} \mathrm{~cm}^{-2}$ (Turner \& Sano 2008) while the cosmic rays' $e$-folding depth is $96 \mathrm{~g} \mathrm{~cm}^{-2}$ (Umebayashi \& Nakano 1981, 2009). The radionuclide abundances correspond to the interstellar dust-to-gas mass ratio $\epsilon=0.01$.

over the upper hemisphere and their secondary particles are absorbed over a column $96 \mathrm{~g} \mathrm{~cm}^{-2}$ following Umebayashi \& Nakano (1981, 2009).

We consider two radioisotope ionization scenarios. Longlived isotopes such as potassium-40 yield an ionization rate $6.9 \times 10^{-23}(\epsilon / 0.01) \mathrm{s}^{-1}$, while short-lived isotopes such as aluminum-26 if present yield a much higher rate, $3.7 \times$ $10^{-19}(\epsilon / 0.01)$, where $\epsilon$ is the dust-to-gas mass ratio (Stepinski 1992; Takata \& Stevenson 1996; Umebayashi \& Nakano 2009; Castillo-Rogez et al. 2009).

The solar nebula for most of its lifetime blocks direct sightlines so that the protosolar X-rays reach the planet's vicinity entirely through scattering. Jupiter and its disk at one time lay in a gap in the solar nebula (Lin \& Papaloizou 1993) and the geometry of the gap surely influenced the flux of X-rays reaching the planet. Furthermore, toward the end of the solar nebula's evolution the gas interior to Jupiter's orbit cleared first, judging from the central holes observed in the so-called transitional systems found among protostellar disks today (Calvet et al. 2005; Muzerolle et al. 2010; Andrews et al. 2011). Jupiter and surrounding material were then directly exposed to protosolar $\mathrm{X}$-rays. However, lacking detailed information about the X-ray transfer in either of these geometries, we use the ionization rates versus column in the solar nebula derived from Monte Carlo transfer calculations by Igea \& Glassgold (1999), taking the case with the $5 \mathrm{keV}$ thermal spectrum from their Figure 3 and scaling the luminosity to $2 \times 10^{30} \mathrm{erg} \mathrm{s}^{-1}$, the median observed in young solar-mass stars in the Orion Nebula Cluster (Garmire et al. 2000). The scattered X-rays are absorbed in a column of about $8 \mathrm{~g} \mathrm{~cm}^{-2}$.

The ionization rate contributions from all the non-thermal processes are shown as functions of the mass column in Figure 2.

Finally, we treat the thermal ionization of the low-ionizationpotential element potassium, which becomes important at the temperatures above $1000 \mathrm{~K}$ reached inside Io's orbit (Takata \& Stevenson 1996). We solve the Saha equation using potassium's 
ionization energy of $4.3407 \mathrm{eV}$ and assuming $99 \%$ of the solar potassium abundance is locked up inside the grains, with the remaining $1 \%$ in the gas and available for collisional ionization. The steep temperature dependence of the thermal ionization means the dead zone boundary is insensitive to this choice.

\section{MAGNETO-ROTATIONAL TURBULENCE}

Analytic and numerical results indicate that the criterion for magneto-rotational instability to drive turbulence is

$$
\Lambda \equiv \frac{v_{A z}^{2}}{\eta \Omega}>1,
$$

where the dimensionless Elsasser number $\Lambda$ depends on the Alfvén speed $v_{\mathrm{Az}}$ for the vertical component of the magnetic field, along with the magnetic diffusivity $\eta$ and orbital frequency $\Omega$. This means the instability must grow faster than the magnetic fields can diffuse across its fastest-growing wavelength (Jin 1996; Sano \& Miyama 1999; Sano \& Inutsuka 2001; Sano \& Stone 2002; Turner et al. 2007). In the diffusivity we include the contributions from the induction equation's Ohmic and ambipolar terms, added in quadrature. A further requirement for the instability to grow near its top rate is that the background toroidal magnetic fields have a pressure less than the gas pressure (Kim \& Ostriker 2000).

We compute the diffusivity $\eta$ including the current densities from all the charged species in the chemical network described in Section 4, following Equations (21)-(31) of Wardle (2007). Both the Ohmic and ambipolar terms in the induction equation are included. Ohmic diffusion occurs at densities high enough for the main charged species to couple to the neutrals through collisions, while ambipolar drift is important when densities are low enough and collisions rare enough that the neutrals slip through the plasma which remains tied to the magnetic fields by Lorentz forces. At intermediate densities a third nonideal effect, the Hall term, is important (Wardle \& Ng 1999). We neglect the Hall term because it affects the turbulence threshold and saturation level only slightly when comparable to the Ohmic term (Sano \& Stone 2002). However, dramatic effects appear in unstratified non-linear calculations when the Hall term dominates (Kunz \& Lesur 2013). Stratified calculations are urgently needed.

The maximum possible accretion stress depends on the Elsasser number $\Lambda$. When the diffusivity is dominated by the ambipolar term, the stress can reach about $1 \%$ of the gas pressure if $\Lambda \approx 1$, and $10 \%$ if $\Lambda \approx 10$, according to three-dimensional unstratified shearing-box MHD results (Bai \& Stone 2011). The higher of these stress levels can occur starting one decade above our magnetic activity threshold, or one contour level in the plots in Section 6 below.

\subsection{Magnetic Fields}

The magneto-rotational instability grows from initially weak magnetic fields into long-lived turbulence in both local and global MHD calculations (Miller \& Stone 2000; Fromang \& Nelson 2006). Over a wide range of seed field strengths, the pressure in the fields' vertical component saturates between $10^{-4}$ and $10^{-2}$ times the midplane gas pressure (Miller \& Stone 2000; Fromang \& Nelson 2006; Shi et al. 2010; Flaig et al. 2010; Okuzumi \& Hirose 2011). Owing to the fields' buoyancy, the magnetic pressure declines more slowly with height than the gas pressure. We therefore compute the Elsasser number at each point assuming that the pressure in the vertical component of the magnetic field is simply $0.1 \%$ of the midplane gas pressure, independent of height. Note that this measures not just the net vertical or seed magnetic field delivered with the gas arriving from the solar nebula, but the overall vertical field including the part generated locally in the turbulence. We seek places in the circumjovian disk where turbulence can be sustained.

Choosing $10^{-3}$ for the midplane pressure ratio means the magnetic field's vertical component has pressure greater than the gas above 3.7 density scale heights. In saturated MRI turbulence, the toroidal magnetic field has a pressure at least 10 times the vertical component (Miller \& Stone 2000), giving a total magnetic pressure exceeding the gas pressure above about $3 H$. The MRI's linear growth rate is reduced at low plasma beta (Kim \& Ostriker 2000) so turbulence would be increasingly weaker above this height. However there would be less weakening if we included (1) the field strength's fall-off above a few scaleheights, and (2) the gas pressure profile's extended tail resulting from magnetic support. Both these effects increase the plasma beta over our simple picture, and both are observed in the threedimensional numerical calculations cited above.

Jupiter's magnetic field can safely be neglected since it is weaker than the MRI-generated fields in all our disks. This is true if the planet's field strength is 10 Gauss at its surface, located at $2 R_{J}$, and falls off like a dipole in proportion to the inverse cube of the radius.

\section{DEAD ZONES}

\subsection{Tests}

As a test, we begin by replicating the Takata \& Stevenson (1996) findings under ionization by short-lived radioisotopes and cosmic rays. Their gas-phase reaction network differs from ours in lacking charge transfer to metal atoms, including instead charge transfer to methane, ammonia and water molecules. They consider grains $1 \mathrm{~cm}$ in radius, which contribute negligibly to the overall recombination cross-section. Our network can be made very like theirs by removing the dust and replacing the magnesium with a generic molecule having abundance $10^{-3}$ per hydrogen atom and recombination rate coefficient $10^{-6} \mathrm{~cm}^{3} \mathrm{~s}^{-1}$. The resulting ionization fractions in the MM96 model disk closely follow Takata \& Stevenson (1996, Figures 4(b), (c), and (f)).

Next we restore the gas-phase reaction network described in Section 4, including the metal ions which are long-lived due to their much smaller recombination coefficient. The corresponding ionization fractions in the disk interior are about three orders of magnitude greater. This is consistent with the picture in protostellar disks, where the metal atoms play a significant role when the dust abundance is low (Fromang et al. 2002; Dzyurkevich et al. 2013).

\subsection{Fiducial Models}

We then consider the most favorable situation for the MRI, with ionization by $\mathrm{X}$-rays, cosmic rays and short-lived radionuclides, looking at one fiducial model each from the minimummass and gas-starved classes. The minimum-mass MM96 model is shown in Figure 3. With recombination on grains (top panel), the dead zone extends to five scale heights and above, where the low gas densities mean the boundary is set by ambipolar diffusion. Combined with the low plasma beta above $5 \mathrm{H}$, this means MRI turbulence is weak or absent throughout. In contrast, without dust (bottom panel) the dead zone extends only 

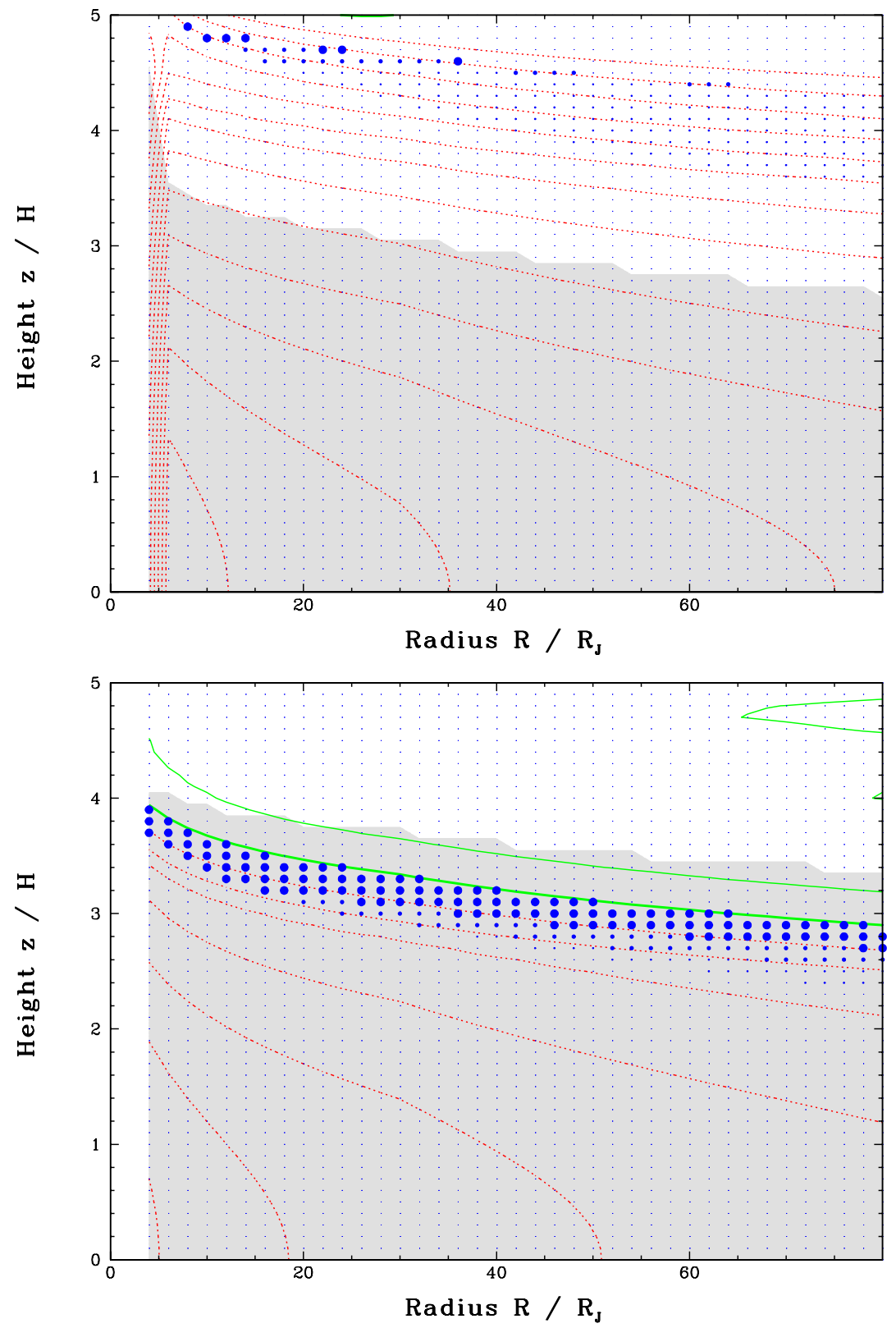

Figure 3. Minimum-mass MM96 model has a substantial dead zone even in the most favorable ionization scenario with X-rays, cosmic rays and short-lived radionuclides. The dusty case is above, the dust-free case below. In the gray shaded region the Ohmic diffusivity is greater than the ambipolar diffusivity. The contours show Elsasser numbers computed from the quadrature sum of the two. The Elsasser number is unity on the heavy green contour. Other contours are spaced by an order of magnitude, with the solid green ones on the MRI-unstable side and the dashed red ones on the MRI-stable side. The uppermost dashed contour in the dusty case is for Elsasser number 0.1. Blue dots mark where turbulent mixing is capable of affecting the dead zone's diffusivity. At the smallest blue dots, the overlying column of free electrons is sufficient to lift the diffusivity above the threshold for turbulence if instantaneously well-mixed. At the medium blue dots, the recombination time is also at least $10 \%$ of the turbulent mixing time. At the largest blue dots, recombination is slower than mixing.

(A color version of this figure is available in the online journal.)

up to $3 H$, leaving a small fraction of the mass column magnetically active. The active layer's lower boundary is set by Ohmic diffusion over its whole length.

The two recombination scenarios shown in Figure 3 yield similar outcomes when applied to the steeper surface density profile $\Sigma=10^{7}\left(R_{J} / R\right)^{1.3} \mathrm{~g} \mathrm{~cm}^{-2}$ originally suggested by Lunine \& Stevenson (1982).

As a fiducial gas-starved disk we choose the K-4 model shown in Figure 4. In contrast to the fiducial minimum-mass model, the disk here resembles the solar nebula in having a substantial magnetically active surface layer overlying an interior dead zone. Over almost the whole radial extent of our calculation, the boundary between the two layers lies below the height of
$3 H$ where the plasma beta falls to unity. MRI can therefore grow at near its maximum rate. Over a similar radial range, the Ohmic term dominates the magnetic diffusivity at the boundary. Basically the whole mass column is active beyond $67 R_{J}$ with dust, or $48 R_{J}$ without.

\subsection{Turbulent Mixing}

We also consider turbulent mixing, which alters the resistivity if the mixing is faster than the chemical reactions. In geometrically thin accretion disks, vertical gradients are generally steeper than radial gradients, so the greatest effects come from mixing in the vertical direction. Representing the mixing as a diffusion process, we can write the mixing time as the ratio of the squared 

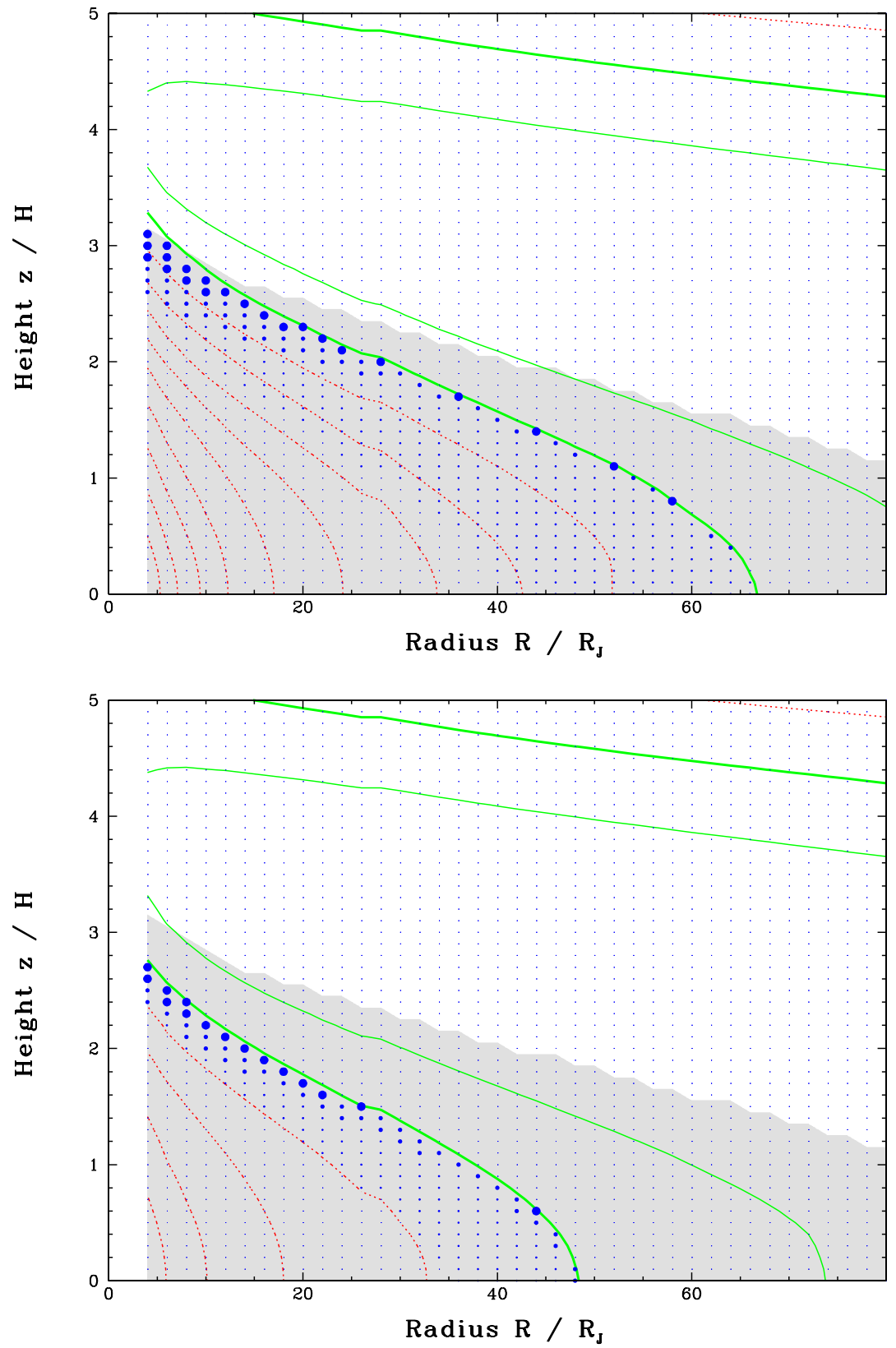

Figure 4. Magnetically active layer and dead zone in the gas-starved K-4 model with ionization by X-rays, cosmic rays, and short-lived radionuclides. Recombination on grains is included in the top panel only. Contours, shading, and symbols are as in Figure 3.

(A color version of this figure is available in the online journal.)

density scale height to the diffusion coefficient. The diffusion coefficient in MRI turbulence is approximately the mean-squared velocity dispersion divided by the shear rate $(3 / 2) \Omega$, and the velocities are roughly equal to the Alfvén speeds. The vertical mixing timescale is therefore

$$
t_{\text {mix }}=\frac{3}{8 \pi}\left(\frac{2 c^{2}}{v_{A z}^{2}}\right) \frac{2 \pi}{\Omega} .
$$

Using the result that MRI turbulence leads to tangled magnetic fields in which the vertical component contributes around $10 \%$ of the magnetic pressure (Miller \& Stone 2000), we can say that the number of orbits needed to mix through one scale height is about equal to the plasma beta parameter $\beta=2 c^{2} / v_{A}^{2}$ for the total magnetic field.
This result lets us estimate the importance of mixing relative to recombination in the circumjovian disk, compared with the nearby solar nebula. The ratio of the turbulent mixing timescale $\sim \beta / \Omega$ to the recombination timescale $\sim 1 / \rho^{2}$ is greater in the small disk in proportion to $\rho^{2} / \Omega \sim(\Sigma / H)^{2} / \Omega \sim$ $\left(1 / 10^{-3}\right)^{2} / 10^{3}=1000$. That is, mixing is less effective in the circumjovian disk. Here we obtained a lower bound by using a surface density $\sim 100 \mathrm{~g} \mathrm{~cm}^{-2}$ from the gas-starved disks, where recombination is slowest among the circumplanetary models. Also, we took similar chemical compositions so that recombination rates are simply proportional to density squared, and we assumed that the MRI turbulence saturates at similar plasma beta values in the two situations, giving comparable mixing timescales when measured in local orbits.

For a more thorough evaluation taking into account the different chemical composition, consider a blob of gas high 

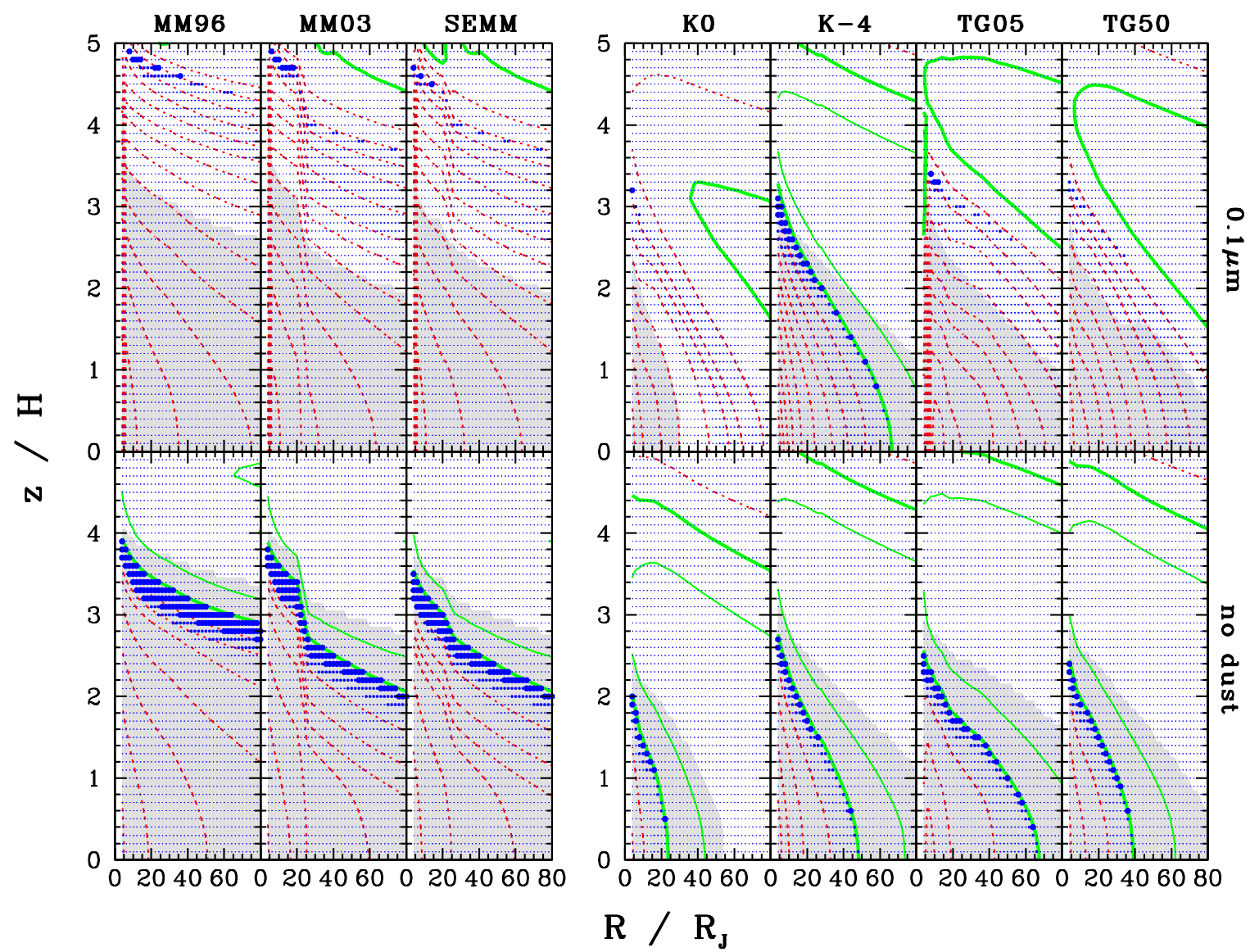

Figure 5. Magnetically active layers and dead zones under ionization by X-rays, cosmic rays, and short-lived radionuclides, in the three minimum-mass (left side) and four gas-starved Jovian subnebula models (right side), with and without recombination on dust (top and bottom rows, respectively). Symbols are as in Figure 3.

(A color version of this figure is available in the online journal.)

in the disk atmosphere where the ionization is substantial. The question is whether, as the blob is carried downward in the turbulence, the electron fraction remains greater than the ambient equilibrium value. To answer, we approximate the reacting flow by moving the blob instantaneously to the interior point of interest. If recombination brings the ionization fraction to equilibrium at the new location over a time at least comparable to the turbulent mixing time, then the mixing can change the ionization state. Sometimes we can avoid even the complication of integrating the chemical network. If mixing the overlying column thoroughly and instantaneously would yield a magnetic diffusivity too high for MRI turbulence, the point of interest will remain dead. We focus on the electrons' mixing, since the transport shifts only the turbulent layer's bottom boundary, which typically is set by the Ohmic diffusivity, and the Ohmic term is controlled by the electron fraction. The procedure in detail is as follows.

1. Determine the local chemical equilibrium ionization states at all heights in some annulus of the model disk.

2. Consider a height $z_{1}$ where the electron fraction is below the MRI threshold or critical value $x_{c}\left(z_{1}\right)$. In local chemical equilibrium, the point $z_{1}$ is magnetically dead. Integrate the columns of free electrons and of neutrals above $z_{1}$ to find the lowest height $z_{2}>z_{1}$ such that the mean electron fraction between $z_{1}$ and $z_{2}$ exceeds the critical value $x_{c}$. If no $z_{2}$ within the model disk yields a mean electron fraction exceeding the critical value, then even instantaneous mixing would not be effective, and point $z_{1}$ will remain dead.
3. Take the abundances of all species $j$ from $z_{2}$ and insert them at $z_{1}$. That is, the number density $n_{1 j}$ is equal to $n_{2 j}\left(n_{1 n} / n_{2 n}\right)$, where the subscripts $n$ indicate the background neutral component. Find the recombination time by integrating the reaction network at $z_{1}$ till the electron fraction drops below the critical value $x_{c}$.

4. The mixing time between the two heights is $\left(z_{2}-z_{1}\right)^{2} / D$, where $D=v_{A z}^{2} / \Omega$ is the turbulent diffusion coefficient measured at $z_{1}$. The diffusion coefficient generally increases with height, so the value at the bottom determines the mixing timescale.

5. Mixing can alter the ionization fraction at $z_{1}$ if recombination is not much faster than mixing. We require $t_{\text {rec }}>$ $0.1 t_{\text {mix }}$.

Similar results come from extending step 2 to consider all heights, and not just the lowest point $z_{2}$ having $x_{e, \text { avg }}>x_{e, c}$. This is because the biggest ratio of recombination to mixing time typically is found at or just above $z_{2}$.

\subsection{Parameter Survey}

Putting together the turbulence criterion and the mixing timescale, we infer that the magnetic fields drive turbulence where either (1) the equilibrium ionization is strong enough by Equation (40), or (2) MRI occurs in the overlying layers in the equilibrium ionization state, and the resulting mixing is fast enough to raise local ionization levels to the threshold. Below we calculate the extent of the turbulence for several models of the Jovian subnebula. 

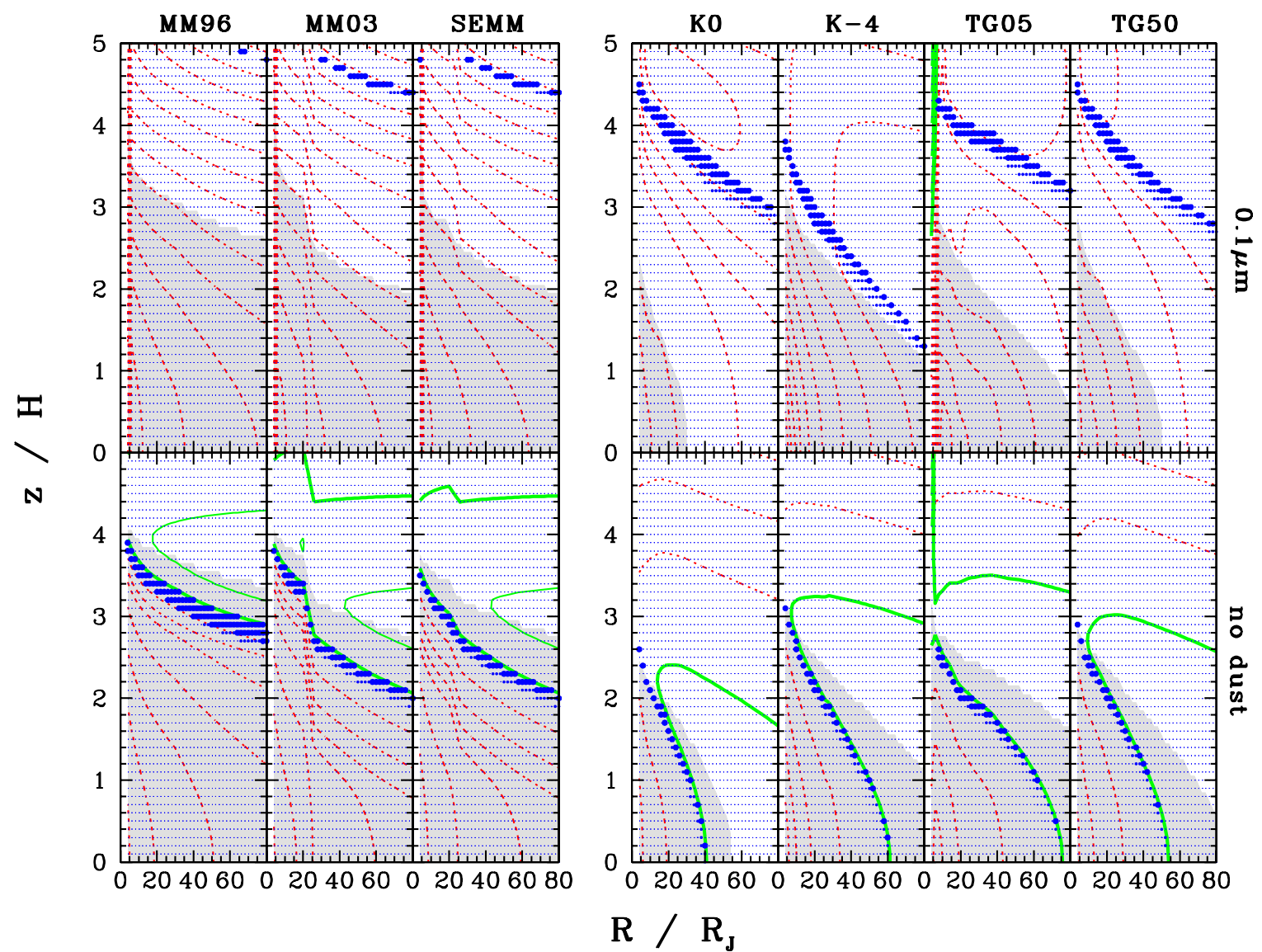

Figure 6. As Figure 5 except that the X-ray ionization is omitted.

(A color version of this figure is available in the online journal.)

The seven circumjovian disk models from Sections 2 and 3 are shown together with their dust-free versions in the next three figures. Each figure corresponds to one ionization scenario. Figure 5 has $\mathrm{X}$-rays, cosmic rays and short-lived radionuclides. Figure 6 has the X-rays switched off. In Figure 7 the X-rays are restored, while the cosmic rays are switched off. The first of these three scenarios is most favorable for magnetic activity, the second is relevant if no protosolar X-rays reach the solar nebula gap where Jupiter and its disk reside (as assumed by Fujii et al. 2011), and the third covers the possibility that the wind from the young Sun screens out the $0.1-1 \mathrm{GeV}$ cosmic rays contributing most to the ionization (Perez-Becker \& Chiang 2011). Each figure has 14 panels, corresponding to the seven model disks with and without dust. In Figure 5 the left top and bottom panels are identical to Figure 3, while the fifth panels in the top and bottom rows reiterate Figure 4. Our calculations extend from $4 R_{J}$ inside Io's present orbit out to $80 R_{J}$ or a little more than $10 \%$ of the planet's Hill radius, and from the equatorial plane up to $5 H$.

In interpreting these figures, recall that X-rays ionize the uppermost few tens of grams per square centimeter, the cosmic rays the next few hundred and the radionuclides dominate in the deeper interior (Figures 1 and 2).

Comparing Figures 5 and 6 we see that the X-ray ionization is crucial for magnetic activity. With X-rays, all gas-starved models show activity at least in their outer reaches. Without $\mathrm{X}$-rays, none of the seven dusty models has a green contour with the sole exception of TG05, where temperatures near $4 R_{J}$ are high enough for thermal ionization. Removing the
$\mathrm{X}$-rays greatly reduces the ion density near the disk surface, pushing down the active layer's ambipolar-diffusion-dominated top boundary in many cases so far that it meets the bottom boundary and the activity is cut off completely. Note that since the blue dots show turbulent mixing's effects on the Ohmic resistivity, the dots are offset from the dead zone edges in locations where those edges are determined by ambipolar diffusion. Mixing is effective only in locations where the blue dots are adjacent to layers subject to MRI turbulence.

In contrast, the cosmic rays have little effect on the dead zone's size. Figure 7 differs from Figure 5 mostly in having more contours in the disks' interiors, indicating the dead zones are more thoroughly dead. As Fujii et al. (2011) found, cosmic rays by themselves generally provide too little ionization to support the MRI in the circumjovian disk. An exception is the minimum-mass models with no dust. Here the dead zone boundary lies near the cosmic ray penetration depth, and is shifted upward a few tenths of a scale height with the cosmic rays excluded.

We also experimented with replacing the short-lived radionuclides by the less-ionizing long-lived radionuclides (not shown). The minimum-mass models' deep interiors are even more diffusive, but the active layer boundaries, with ionization controlled by X-rays or cosmic rays, are unaffected.

Considering the three Figures 5-7 together, we see that in all cases with substantial activity (i.e., where the Elsasser number exceeds ten somewhere) the active layer's bottom boundary lies in or near the Ohmic-dominated region. On the other hand, in all cases where the active layer has an upper boundary lying on 


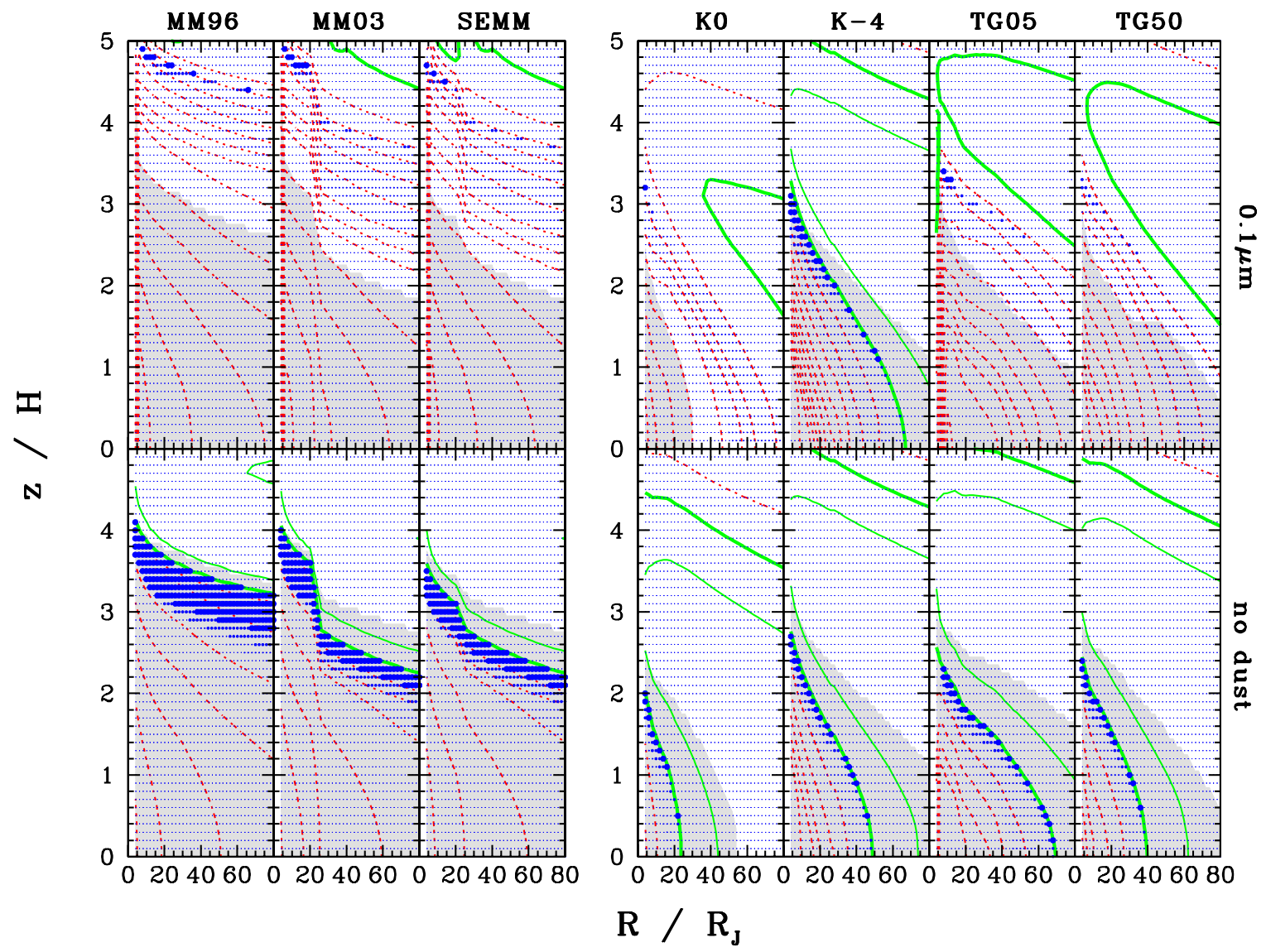

Figure 7. As Figure 5 except that the cosmic ray ionization is omitted.

(A color version of this figure is available in the online journal.)

our grid, that boundary is set by ambipolar diffusion. The upper boundary in many cases also experiences slow MRI growth because it lies above the heights of 3 and $3.7 \mathrm{H}$ where the total and vertical plasma beta fall to unity.

Again considering the three figures together, we see that local chemical equilibrium is mostly a good approximation. Mixing is capable of changing the dead zone boundary by only a few tenths of a scale height. The thickest mixing layer occurs in the MM96 model without dust, where X-ray ionization yields enough free electrons to activate the MRI a half-scale-height into the equilibrium dead zone.

Looking separately at the minimum-mass models, we see that all with dust are quite dead. Magnetic activity is possible only in a low-surface-density zone outside the orbit of Callisto like that advocated by Mosqueira \& Estrada (2003a). However, the dust-free versions of the three minimum-mass models all have more substantial MRI-unstable upper atmospheres. Far from the planet these even extend below $3 H$, where the gas pressure exceeds the magnetic pressure.

The situation is quite different in the gas-starved models, which have an active layer in every scenario with X-rays. The dead zone's size varies among the dusty gas-starved models owing to the differing dust abundances and surface densities. The dusty K0 model is MRI-stable near the planet due to ambipolar diffusion associated with its low densities, while the dusty K-4 model's active layers comfortably reach the planet if $\mathrm{X}$-rays are included. The TG50 model has a similar gas surface density to the $\mathrm{K}-4$, but 100 times greater dust abundance and thus a smaller active region. The TG05 model, with its stronger accretion heating due to a higher mass flow rate, is hot enough inside $6 R_{J}$ for collisional ionization in a surface layer. The results are otherwise insensitive to the details of the temperature and density structure: the dead zones are little-changed when we make the gas-starved disks vertically isothermal at the accretion temperature.

\subsection{Magnetic Field Strength}

Other choices for the magnetic field strength will change the picture as follows. The Ohmic diffusivity is independent of the field strength, while the ambipolar diffusivity is generally proportional to the magnetic pressure (Wardle 2007). The Elsasser number, which by Equation (40) depends on the ratio of the magnetic pressure to the diffusivity, thus varies in proportion to the magnetic pressure if the Ohmic term dominates, and is field-strength-independent in the ambipolar regime.

As an example, if the vertical magnetic field has a pressure 10 times greater than we assumed above, reaching $1 \%$ of the midplane gas pressure, then (1) where the Ohmic term dominates, the Elsasser number is an order of magnitude larger. The circumplanetary disk's interior is better-coupled. (2) Where the ambipolar term dominates, the Elsasser numbers are unchanged. The active layer's top edge typically does not move. (3) The Ohmic-to-ambipolar transition shifts deeper by about one contour. In some cases the dead zone's lower boundary switches from the Ohmic to the ambipolar regime.

\section{SOLID MATERIAL}

In this section we discuss the implications of the circumjovian disk's magnetic activity for the evolution of the solid material 

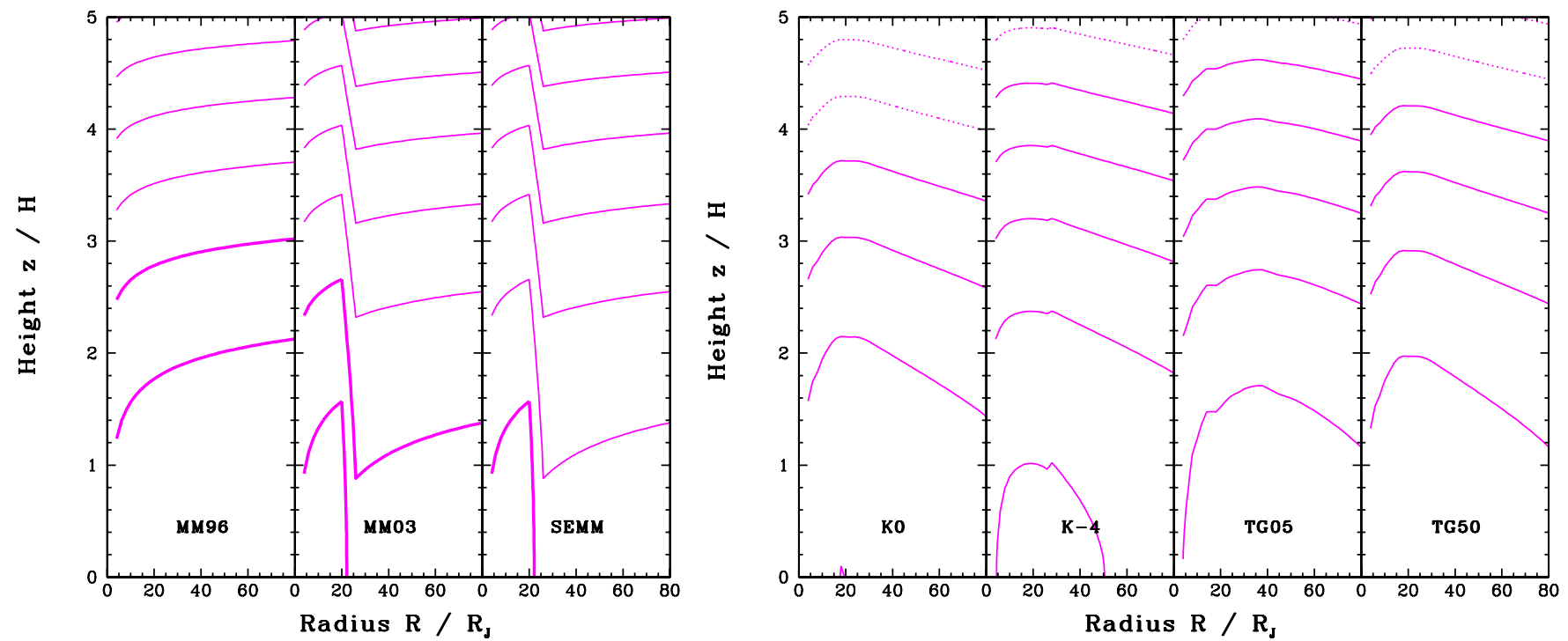

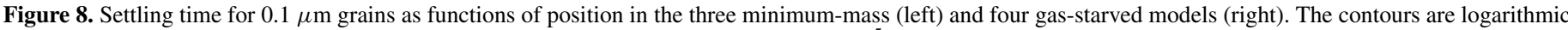

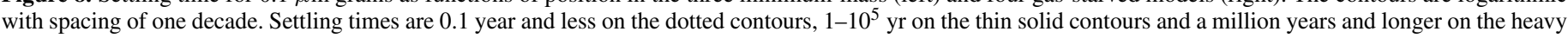
solid contours.

(A color version of this figure is available in the online journal.)

inside. The abundance of solids in the material delivered to the disk could be less than suggested by Jupiter's overall heavy-element abundance of about three times solar (Young 2003). The nearby solar nebula may have become depleted in solids during the assembly of the planet's core (Hubickyj et al. 2005). Also, once the planet grows massive enough to open a gap in the solar nebula, only the fraction of the solid mass contained in small grains is readily carried to the vicinity of the planet according to hydrodynamical results from Paardekooper (2007). Grains smaller than about $10 \mu \mathrm{m}$ are accreted on the planet and its circumplanetary disk, and particles of intermediate size are captured by gas drag in the pressure maxima immediately inside and outside the planet's orbit. Large bodies become trapped in orbital resonances (Weidenschilling \& Davis 1985).

We saw above that solids in the form of fine dust particles can prevent magneto-rotational turbulence through their large recombination cross-section. Removing the dust can restore turbulence in the layers where the ionizing radiation is absorbed. We would therefore like to know, if turbulence is absent, how long before the dust settles out?

The settling time is the distance to the equatorial plane divided by the grains' terminal speed. In the Epstein regime, where the particles are smaller than the gas molecules' mean free path and drift through the gas slower than the sound speed, the settling time $t_{S}=\left(\Omega^{2} t_{D}\right)^{-1}$ where the gas drag stopping time $t_{D}=\left(\rho_{d} / \rho\right)\left(a / c_{s}\right)$ (e.g., Turner et al. 2010). Recalling that our grains are $a=0.1 \mu \mathrm{m}$ in radius with internal density $\rho_{d}=2 \mathrm{~g} \mathrm{~cm}^{-3}$ we obtain the timescales shown in Figure 8.

In the minimum-mass models (left panels), which are stable against MRI turbulence if dusty, the grains settle below $3 \mathrm{H}$ in under a million years. Note that we have neglected coagulation. Grains that stick together to form compact aggregates on colliding settle still faster (Dullemond \& Dominik 2005). Since the minimum-mass models by construction receive no resupply from outside, it seems likely the atmosphere will become dustdepleted. Good coupling to the fields (Figures 5-7) will then let some of the atmosphere accrete on the planet, leaving the disk overall gas-depleted.
In the gas-starved models (right panels), the settling is so fast that the atmosphere would become dust-free down to $3 H$ in less than $1000 \mathrm{yr}$ - except that in these models, dust and gas are resupplied across much of the disk surface. The atmosphere's dust abundance will therefore be determined by external factors. Near the disk midplane, the dust is resupplied faster than it settles. The resupply time is the disk surface density divided by the incoming mass flux. Resupply is slowest at the inner boundary where its timescale is under $2000 \mathrm{yr}$ in all four gas-starved models-lower than the midplane settling time in all cases. Consider also that good magnetic coupling extends to the midplane in the models' outermost annuli at low dust abundances (Figures 5-7). MRI turbulence can then loft grains from the interior into the atmosphere. The most likely outcome for the gas-starved disks is thus an atmosphere containing some dust overlying an interior with a higher dust-to-gas ratio.

Both minimum-mass and gas-starved models contain layered dead zones where the local MRI cannot drive turbulence. The dead zones provide favorable quiescent environments for growing larger bodies (Gressel et al. 2012) which could eventually be assembled into the regular satellites (Lubow \& Martin 2013).

The Epstein regime applies throughout Figure 8, with two minor exceptions. First, the grains settle at a terminal speed that exceeds the sound speed in a fraction of the topmost scale height in the gas-starved models. This simply means these low-density regions quickly lose their dust. Second, the gas mean free path is less than the grain size at the midplane in the innermost annulus of the MM96 model. This densest point of all in the seven models is dead with or without dust, so the settling time there is irrelevant for our purposes.

\section{SUMMARY AND CONCLUSIONS}

We examined the prospects for magnetic activity in seven different models of the circumjovian disk, spanning a range of surface densities and including both minimum-mass and gasstarved models. The gas-starved models were refined to include temperature-dependent opacities and properly treat annuli of 
low optical depth. For each model we computed the ionization state, treating gas-phase recombination, charge transfer to longlived metal atoms, and adsorption of the free charges on grains. Where grains are present, the last of these is the main recombination channel everywhere that the ionization fraction is low enough for the grains to remain within one or two electrons of neutral.

From the abundances of all the charged species we computed the magnetic diffusivities, including the contributions from both Ohmic diffusion and ambipolar drift. The magnetic forces can drive turbulence if the distance the field diffuses per orbit is less than the fastest-growing wavelength of the MRI-that is, if the dimensionless Elsasser number $\Lambda=v_{A z}^{2} /(\eta \Omega)$ is bigger than unity. To see where magneto-rotational turbulence is possible, we plotted contours of the Elsasser number versus distance from the planet and height above the equatorial plane, including both Ohmic and ambipolar terms in the diffusivity $\eta$ and choosing a magnetic field whose vertical component has a pressure $0.1 \%$ of the midplane gas pressure. We investigated two limiting cases: the stellar X-rays either (1) reach the circumplanetary disk with the same flux as at the corresponding column in the nearby solar nebula, or (2) are blocked completely by the nebula.

All the dusty minimum-mass models we considered are thoroughly magnetically dead, with or without X-ray ionization. MRI turbulence is unlikely to occur near the midplane in the dense, cold parts of a minimum-mass circumjovian disk. However, if turbulence is absent, the dust will settle out of the upper layers in a relatively short time. Removing the dust greatly reduces the recombination rate, allowing MRI turbulence in a surface layer reaching down to near the cosmic ray penetration depth, or if cosmic rays are excluded, the $\mathrm{X}$-ray penetration depth. In particular, surface layer angular momentum transport by magnetic forces is possible in models where the surface density falls off steeply beyond Callisto. These models might need to be modified to include stronger accretion stresses in the outer part. More generally, if the dust-depleted surface layers accrete on the planet or are removed through photoevaporation, the disk will be left gas-poor overall. It seems possible that minimum-mass models turn into something resembling the solids-enhanced minimum-mass models put forward by Mosqueira \& Estrada (2003a, 2003b) and Estrada et al. (2009).

By contrast, all the gas-starved models whether dusty or dust-free have an accreting surface layer, with one group of exceptions: in the dusty cases without X-rays, the ion densities are so low that ambipolar diffusion prevents magnetic fields from acting on the bulk neutral gas. Another consequence of the gas-starved models' low densities is that grains rapidly settle out in the absence of turbulence. A supply of fresh dust and gas from the solar nebula is assumed in constructing these models. The resupply is fast enough to keep the interior dusty, but too slow to prevent settling from partially depleting the atmosphere. Furthermore, we saw that turbulence is capable of reaching the midplane in outer annuli, so small grains can be returned to the atmosphere through mixing. The most likely outcome is an atmosphere with a reduced dust content.

In summary, both minimum-mass and gas-starved models of the circumjovian disk have conductivities generally sufficient for magnetic forces to provide the assumed accretion stresses. However, a key quantity is the X-ray flux reaching the neighborhood of the planet. Without the X-rays, the dusty gas-starved models couple to magnetic fields too poorly for magneto-rotational turbulence to operate. The minimum-mass models' low internal conductivities with or without X-rays, on the other hand, are as required to keep the material in place. Both classes of models can develop layered dead zones, which could provide a favorable quiescent environment for assembling regular satellites (Lubow \& Martin 2013).

The magnetic coupling maps point to several more areas where our understanding is lacking. Future minimum-mass modeling may need to treat the loss of dust-depleted gas from the surface layers. In the gas-starved models, the stress-topressure ratio ought to increase with radius. Consequently, the material will pile up at locations where the inflow slows. Episodic accretion outbursts will result if some additional angular momentum transport process switches on when the disk surface density grows large enough. The trigger can be the gravitational instability if the accretion bottleneck fills up so much that a gas-starved model approaches the surface densities of a minimum-mass model (Lubow \& Martin 2012).

For the future development of the gas-starved models it is important to address the issue of weak magnetic coupling in the absence of stellar X-rays. This motivates more careful calculations of the transfer of the X-rays into the gap opened in the solar nebula by Jupiter's tides. The X-rays could have reached the circumplanetary disk at full strength if the solar nebula interior to the planet's orbit was cleared away, as appears to have happened in the so-called transitional disks observed around some young stars today (Calvet et al. 2005; Muzerolle et al. 2010; Andrews et al. 2011). Also, planets lying nearer their stars can have better-ionized disks owing to the greater X-ray intensities.

Further constraints on the conditions in the circumjovian disk can potentially be derived from the Laplace resonance. The three inner Galilean moons, Io, Europa, and Ganymede, have orbital periods nearly in the ratio 1:2:4. Peale \& Lee (2002), Sasaki et al. (2010), and Ogihara \& Ida (2012) demonstrated that resonances can be assembled outside-in during satellite formation and migration in gas-starved subnebula models. Whether this works in circumjovian disk models with magnetic stresses remains to be seen.

We have focused on the cold parts of the disk. The thermally ionized zone near the planet could be important for its role in regulating the planet's spin (Lovelace et al. 2011), launching bipolar jets (Fendt 2003; Machida et al. 2006), and determining whether the planet begins its life cold or warm (Marley et al. 2007). The strong temperature dependence of the ionization in this regime suggests that better thermodynamical models are needed.

This work was supported by the NASA Outer Planets Research program through grant 07-OPR07-0065, by the Hong Kong Research Grants Council through grant HKU 7024/08P, and by the Center for Planetary Science at Kobe University under the auspices of the MEXT Global COE program titled "Foundation of International Center for Planetary Science." The work was carried out in part at the Jet Propulsion Laboratory, California Institute of Technology.

\section{REFERENCES}

Alibert, Y., Mousis, O., \& Benz, W. 2005, A\&A, 439, 1205

Anderson, J. D., Jacobson, R. A., McElrath, T. P., et al. 2001, Icar, 153, 157

Andrews, S. M., Wilner, D. J., Espaillat, C., et al. 2011, ApJ, 732, 42

Armitage, P. J. 2011, ARA\&A, 49, 195

Ayliffe, B. A., \& Bate, M. R. 2012, MNRAS, 427, 2597

Bai, X.-N. 2011, ApJ, 739, 50 
Bai, X.-N., \& Stone, J. M. 2011, ApJ, 736, 144

Balbus, S. A., \& Hawley, J. F. 1991, ApJ, 376, 214

Balbus, S. A., \& Hawley, J. F. 1998, RvMP, 70, 1

Barr, A. C., \& Canup, R. M. 2008, Icar, 198, 163

Bodenheimer, P. 1995, ARA\&A, 33, 199

Calvet, N., D’Alessio, P., Watson, D. M., et al. 2005, ApJL, 630, L185

Canup, R. M., \& Ward, W. R. 2002, AJ, 124, 3404

Canup, R. M., \& Ward, W. R. 2006, Natur, 441, 834

Canup, R. M., \& Ward, W. R. 2009, in Europa, ed. R. T. Pappalardo, W. B. McKinnon, \& K. K. Khurana (Tucson, AZ: Univ. Arizona Press), 59

Castillo-Rogez, J., Johnson, T. V., Lee, M. H., et al. 2009, Icar, 204, 658

Chiang, E. I., \& Goldreich, P. 1997, ApJ, 490, 368

Crida, A., \& Charnoz, S. 2012, Sci, 338, 1196

Dullemond, C. P., \& Dominik, C. 2005, A\&A, 434, 971

Dzyurkevich, N., Turner, N. J., Henning, T., \& Kley, W. 2013, ApJ, 765, 114

Estrada, P. R., \& Mosqueira, I. 2006, Icar, 181, 486

Estrada, P. R., Mosqueira, I., Lissauer, J. J., D’Angelo, G., \& Cruikshank, D. P. 2009, in Europa, ed. R. T. Pappalardo, W. B. McKinnon, \& K. K. Khurana (Tucson, AZ: Univ. of Arizona Press), 27

Fendt, C. 2003, A\&A, 411, 623

Flaig, M., Kley, W., \& Kissmann, R. 2010, MNRAS, 409, 1297

Fromang, S., \& Nelson, R. P. 2006, A\&A, 457, 343

Fromang, S., Terquem, C., \& Balbus, S. A. 2002, MNRAS, 329, 18

Fujii, Y. I., Okuzumi, S., \& Inutsuka, S.-i. 2011, ApJ, 743, 53

Gao, P., \& Stevenson, D. J. 2013, Icar, 226, 1185

Garmire, G., Feigelson, E. D., Broos, P., et al. 2000, AJ, 120, 1426

Goodman, J., \& Rafikov, R. R. 2001, ApJ, 552, 793

Gressel, O., Nelson, R. P., \& Turner, N. J. 2012, MNRAS, 422, 1140

Hirose, S., \& Turner, N. J. 2011, ApJL, 732, L30

Hubeny, I. 1990, ApJ, 351, 632

Hubickyj, O., Bodenheimer, P., \& Lissauer, J. J. 2005, Icar, 179, 415

Igea, J., \& Glassgold, A. E. 1999, ApJ, 518, 848

Ilgner, M., \& Nelson, R. P. 2006, A\&A, 445, 205

Jin, L. 1996, ApJ, 457, 798

Kim, W.-T., \& Ostriker, E. C. 2000, ApJ, 540, 372

Kunz, M. W., \& Lesur, G. 2013, MNRAS, 434, 2295

Kusaka, T., Nakano, T., \& Hayashi, C. 1970, PThPh, 44, 1580

Li, W. Y., \& Lee, M. H. 2013, Protostars and Planets VI, 97

Lin, D. N. C., \& Papaloizou, J. 1986, ApJ, 307, 395

Lin, D. N. C., \& Papaloizou, J. C. B. 1993, in Protostars and Planets III, ed. E. H. Levy \& J. I. Lunine (Tucson, AZ: Univ. Arizona Press), 749

Lovelace, R. V. E., Covey, K. R., \& Lloyd, J. P. 2011, AJ, 141, 51

Lubow, S. H., \& Martin, R. G. 2012, ApJL, 749, L37

Lubow, S. H., \& Martin, R. G. 2013, MNRAS, 428, 2668

Lubow, S. H., Seibert, M., \& Artymowicz, P. 1999, ApJ, 526, 1001

Lunine, J. I., \& Stevenson, D. J. 1982, Icar, 52, 14
Lynden-Bell, D., \& Pringle, J. E. 1974, MNRAS, 168, 603

Machida, M. N., Inutsuka, S.-i., \& Matsumoto, T. 2006, ApJL, 649, L129

Machida, M. N., Kokubo, E., Inutsuka, S.-i., \& Matsumoto, T. 2008, ApJ, 685,1220

Malbet, F., Lachaume, R., \& Monin, J.-L. 2001, A\&A, 379, 515

Marley, M. S., Fortney, J. J., Hubickyj, O., Bodenheimer, P., \& Lissauer, J. J. 2007, ApJ, 655, 541

Martin, R. G., \& Lubow, S. H. 2011, MNRAS, 413, 1447

McKinnon, W. B. 1997, Icar, 130, 540

Miller, K. A., \& Stone, J. M. 2000, ApJ, 534, 398

Mitchell, T. R., \& Stewart, G. R. 2011, AJ, 142, 168

Mosqueira, I., \& Estrada, P. R. 2003a, Icar, 163, 198

Mosqueira, I., \& Estrada, P. R. 2003b, Icar, 163, 232

Muzerolle, J., Allen, L. E., Megeath, S. T., Hernández, J., \& Gutermuth, R. A. 2010, ApJ, 708, 1107

Ogihara, M., \& Ida, S. 2012, ApJ, 753, 60

Okuzumi, S., \& Hirose, S. 2011, ApJ, 742, 65

Paardekooper, S.-J. 2007, A\&A, 462, 355

Peale, S. J., \& Lee, M. H. 2002, Sci, 298, 593

Perez-Becker, D., \& Chiang, E. 2011, ApJ, 735, 8

Pollack, J. B., Hollenbach, D., Beckwith, S., et al. 1994, ApJ, 421, 615

Pollack, J. B., McKay, C. P., \& Christofferson, B. M. 1985, Icar, 64, 471

Pringle, J. E. 1981, ARA\&A, 19, 137

Rivier, G., Crida, A., Morbidelli, A., \& Brouet, Y. 2012, A\&A, 548, A116

Ruden, S. P., \& Pollack, J. B. 1991, ApJ, 375, 740

Safronov, V. S., Pechernikova, G. V., Ruskol, E. L., Vitjazev, A. V., \& Vityazev,

A. V. 1986, in Satellites, ed. J. A. Burns \& M. S. Matthews (Tucson, AZ: Univ. Arizona Press), 89

Sano, T., \& Inutsuka, S. 2001, ApJL, 561, L179

Sano, T., \& Miyama, S. M. 1999, ApJ, 515, 776

Sano, T., \& Stone, J. M. 2002, ApJ, 577, 534

Sasaki, T., Stewart, G. R., \& Ida, S. 2010, ApJ, 714, 1052

Shakura, N. I., \& Sunyaev, R. A. 1973, A\&A, 24, 337

Shi, J., Krolik, J. H., \& Hirose, S. 2010, ApJ, 708, 1716

Stepinski, T. F. 1992, Icar, 97, 130

Takata, T., \& Stevenson, D. J. 1996, Icar, 123, 404

Tanigawa, T., Ohtsuki, K., \& Machida, M. N. 2012, ApJ, 747, 47

Turner, N. J., Carballido, A., \& Sano, T. 2010, ApJ, 708, 188

Turner, N. J., \& Sano, T. 2008, ApJL, 679, L131

Turner, N. J., Sano, T., \& Dziourkevitch, N. 2007, ApJ, 659, 729

Umebayashi, T., \& Nakano, T. 1981, PASJ, 33, 617

Umebayashi, T., \& Nakano, T. 2009, ApJ, 690, 69

Wardle, M. 2007, Ap\&SS, 311, 35

Wardle, M., \& Ng, C. 1999, MNRAS, 303, 239

Weidenschilling, S. J., \& Davis, D. R. 1985, Icar, 62, 16

Young, R. E. 2003, NewAR, 47, 1 